Article

\title{
Physical Gold Nanoparticle-Decorated Polyethylene Glycol-Hydroxyapatite Composites Guide Osteogenesis and Angiogenesis of Mesenchymal Stem Cells
}

\author{
Chiung-Chyi Shen ${ }^{1,2,3}$, Shan-hui Hsu ${ }^{4}{ }^{(0)}$, Kai-Bo Chang ${ }^{5}$, Chun-An Yeh ${ }^{5}$, Hsiang-Chun Chang ${ }^{5}$, \\ Cheng-Ming Tang ${ }^{6}{ }^{(}$, Yi-Chin Yang ${ }^{1}{ }^{(D)}$, Hsien-Hsu Hsieh ${ }^{7}\left(\mathbb{D}\right.$ and Huey-Shan Hung ${ }^{5,8, *(D)}$
}

check for

updates

Citation: Shen, C.-C.; Hsu, S.-h.; Chang, K.-B.; Yeh, C.-A.; Chang, H.-C.; Tang, C.-M.; Yang, Y.-C.; Hsieh, H.-H.; Hung, H.-S. Physical Gold Nanoparticle-Decorated Polyethylene Glycol-Hydroxyapatite Composites Guide Osteogenesis and Angiogenesis of Mesenchymal Stem Cells. Biomedicines 2021, 9, 1632. https:// doi.org/10.3390/biomedicines9111632

Academic Editor: José García Solé

Received: 2 October 2021

Accepted: 4 November 2021

Published: 6 November 2021

Publisher's Note: MDPI stays neutral with regard to jurisdictional claims in published maps and institutional affiliations.

Copyright: (c) 2021 by the authors. Licensee MDPI, Basel, Switzerland. This article is an open access article distributed under the terms and conditions of the Creative Commons Attribution (CC BY) license (https:// creativecommons.org/licenses/by/ $4.0 /)$.
1 Department of Neurosurgery, Neurological Institute, Taichung Veterans General Hospital, Taichung 407204, Taiwan; shengeorge@yahoo.com (C.-C.S.); jean1007@gmail.com (Y.-C.Y.)

2 Department of Physical Therapy, Hung Kuang University, Taichung 433304, Taiwan

3 Basic Medical Education Center, Central Taiwan University of Science and Technology, Taichung 406053, Taiwan

4 Institute of Polymer Science and Engineering, National Taiwan University, Taipei 10617, Taiwan; shhsu@ntu.edu.tw

5 Graduate Institute of Biomedical Science, China Medical University, Taichung 40402, Taiwan; kbwork2021@gmail.com (K.-B.C.); fireleafmaple@hotmail.com (C.-A.Y.); hs0603@gmail.com (H.-C.C.)

6 College of Oral Medicine, Chung Shan Medical University, Taichung 40201, Taiwan; ranger@csmu.edu.tw

7 Blood Bank, Taichung Veterans General Hospital, Taichung 407204, Taiwan; hhhsu@vghtc.gov.tw

8 Translational Medicine Research, China Medical University Hospital, Taichung 40402, Taiwan

* Correspondence: hunghs@mail.cmu.edu.tw; Tel.: +886-4-2205-2121 (ext. 7827); Fax: +886-4-2233-3641

Abstract: In this study, polyethylene glycol (PEG) with hydroxyapatite (HA), with the incorporation of physical gold nanoparticles (AuNPs), was created and equipped through a surface coating technique in order to form PEG-HA-AuNP nanocomposites. The surface morphology and chemical composition were characterized using scanning electron microscopy (SEM), atomic force microscopy (AFM), UV-Vis spectroscopy (UV-Vis), Fourier transform infrared spectroscopy (FTIR), X-ray photoelectron spectroscopy (XPS), and contact angle assessment. The effects of PEG-HA-AuNP nanocomposites on the biocompatibility and biological activity of MC3T3-E1 osteoblast cells, endothelial cells (EC), macrophages (RAW 264.7), and human mesenchymal stem cells (MSCs), as well as the guiding of osteogenic differentiation, were estimated through the use of an in vitro assay. Moreover, the anti-inflammatory, biocompatibility, and endothelialization capacities were further assessed through in vivo evaluation. The PEG-HA-AuNP nanocomposites showed superior biological properties and biocompatibility capacity for cell behavior in both MC3T3-E1 cells and MSCs. These biological events surrounding the cells could be associated with the activation of adhesion, proliferation, migration, and differentiation processes on the PEG-HA-AuNP nanocomposites. Indeed, the induction of the osteogenic differentiation of MSCs by PEG-HA-AuNP nanocomposites and enhanced mineralization activity were also evidenced in this study. Moreover, from the in vivo assay, we further found that PEG-HA-AuNP nanocomposites not only facilitate the anti-immune response, as well as reducing CD86 expression, but also facilitate the endothelialization ability, as well as promoting CD31 expression, when implanted into rats subcutaneously for a period of 1 month. The current research illustrates the potential of PEG-HA-AuNP nanocomposites when used in combination with MSCs for the regeneration of bone tissue, with their nanotopography being employed as an applicable surface modification approach for the fabrication of biomaterials.

Keywords: polyethylene glycol; hydroxyapatite; physical gold nanoparticle; mesenchymal stem cells

\section{Introduction}

According to recent studies, poly (lactic-co-glycolic acid) (PLGA)-based artificial bonesubstitute materials are highly attractive due to their better biocompatibility, degradability, 
and mechanical properties which enhance bone regeneration [1,2]. A hydrogel-based drug delivery system combined with nanoparticles has been used as a drug reservoir in order to improve the treatment effect [3]. Stem cells have been differentiated into chondrocytes with the assistance of either chondrogenic factors or scaffolds [4]. Moreover, a previous study indicated that scaffolds and growth factors are two important components that influence bone regeneration. PLGA/HA composite scaffolds with controlled gene release and Dox-regulated gene expression by electrical stimulation promoted cell proliferation and osteogenic differentiation in vitro while enhancing bone repair in an in vivo rabbit radial defect model [5].

A perfect biomaterial for bone substitution should be biocompatible, biodegradable, and osteoconductive [6]. Currently, different types of bone biomaterials including ceramics, polymers, decalcified matrices, bioactive glasses, hydroxyapatite (HA), and collagen (Col) have been widely applied for use as essential components for bone tissue regeneration [7-10]. Additionally, certain principal polymers, such as polyesters, poly (L-lactic acid) (PLA), poly (glycolide) (PGA), and poly (lactide-co-glycolide) (PLGA), have also been extensively certified due to their superior mechanical and physical properties for bone tissue regeneration [11,12]. However, the lack of sufficient biological activity in these polymers implies they will still encounter limitations. For example, HA ceramics have a disadvantage due to their poor hydrophilic properties for bone tissue regeneration [13]. For this reason, integration involving the advantages of bone biomaterials offers a new strategy towards ameliorating biomimic functions and mechanical properties and remains a critical issue for now.

Hydroxyapatite is the main mineral component in bone tissue. HA has a distinct surface effect, meaning it is prevalent in a wide range of biological molecules, such as proteins or growth factors $[14,15]$. Therefore, in order to achieve the application of the HA polymer, appropriate interfacial properties between the polymer and mineral components are required in order to accomplish mechanical, chemical, and biological capabilities [16,17]. In addition, the inherent property of HA that encourages osteoblast or mesenchymal stem cell recruitment and growth leads to osteogenesis [18,19]. Recently, the HA polymer has been widely applied as a replacement bone material, including HA-PLA [20], collagen [21], polyamides [22], poly $\alpha$-caprolactone [23], and poly ( $\alpha$-hydroxy acid) [24]. A report indicated that HA-PLGA nanocomposites are created by gas formation and particle leaching methods with 3D fiber deposition, which can promote bone regeneration [6]. Another study also demonstrated that crosslinked poly ( $\varepsilon$-caprolactone) (PCL) administers iron into hydroxyapatite (Fe-HA) nanoparticles to form 3D PCL/Fe-HA scaffolds, which can prompt the mechanical and mass transfer of properties for bone tissue repair, as evaluated in a rabbit model [25]. However, the remaining 3D PCL/Fe-HA scaffolds encountered lower uniformity and poor mechanical properties. Additionally, chitosancoated iron oxide nanoparticles enhanced osteoblast proliferation, decreased cell membrane damage, and promoted cell differentiation. These works indicate that the presence of organic/inorganic nanoparticles can promote osteogenic differentiation [26]. The incorporation of HA nanoparticles into a poly(ethylene glycol) (PEG)-based hydrogel to guide its mineralization potentiality has been well elucidated for bone tissue regeneration [27]. A previous study demonstrated the effects of cellulose nanofibers (CNFs) and hydroxyapatite (HA) nanoparticles incorporated into a poly( $\varepsilon$-caprolactone) (PCL) matrix. The surface wettability and mechanical properties of PCL scaffolds were improved by CNFs, and further with HA addition. The results of the compressive and elastic moduli also verified the excellent properties for bone tissue engineering [28]. Furthermore, the addition of HA and CNFs did not weaken the biocompatibility of PCL/CNF/HA nanocomposites [28]. Moreover, crystalline HA has been proved to be biocompatible and osteoconductive, and it exhibited the slowest degradation rate compared with other calcium phosphates [29].

Modification of the functional properties (e.g., viscoelasticity, modulus, permeability, and injectability features) of nanocomposite hydrogels/gels has been employed for bone tissue regeneration [30-32]. Previous studies were also dedicated to developing 3D 
nanocomposite poly hydroxyapatite scaffolds for bone tissue regeneration [33]. In line with other studies, injectable hydroxyapatite hydrogel composites offered a better performance in guided bone regeneration $[34,35]$. A fibrous scaffold incorporated within a PEG hydrogel was shown to improve the mechanical properties [36]. In addition, a study revealed that collagen/n-HA hydrogels promoted spinal fusion [37]. A previous study evaluated the osteogenesis of bone marrow-derived human mesenchymal stem cells (hMSCs) with an implanted PEG-HA scaffold by an in vivo assay, showing that collagen deposition in the PEG-HA scaffold was prominent [8]. Additionally, a synthetic hydroxyapatite/ $\beta$-tricalcium phosphate (HA/TCP) material blended with a PEG hydrogel, including a blend with human Bone Morphogenetic Proteins-2 (rhBMP-2), enhanced new bone tissue formation as compared to BMP-2 delivered using the HA/TCP construct alone [38].

Gold nanoparticles (AuNPs) offer a promising multi-angled approach for tissue regeneration [39]. AuNPs can easily be contoured to different sizes and shapes which can then be manufactured by surface plasmon resonance (SPR), a phenomenon involving the interaction between the electromagnetic wave and the conduction electrons in materials [40] which includes cytotoxicity, uptake, biodistribution, and pharmacokinetics [41]. As discussed in our previous reports, AuNPs have a distinct property which can affect many types of polymers such as polyurethane, fibronectin, and collagen via their interaction with the polymer to form the nanotopography, as well as the ability to display superior biological performance, including modifying cell behavior which is specific for induction involving the differentiation capability of stem cells [42-44]. AuNPs are appropriate components for the functionalization of electrospun scaffolds for bone regeneration, due to their properties of encouraging osteogenic differentiation in stem cells and osteoclasts [45-48]. The binding of AuNPs is specific for the surface of HA with its high binding affinity to HA [49]. Another report also confirmed that AuNPs can notably promote the osteogenic process in calvaria animal defect models [50]. Therefore, it has also been well established that AuNPs are potential nanoparticles for osteogenesis. Furthermore, whether AuNPs can enhance MSCs regarding osteogenic differentiation by upregulating the osteogenic gene Runt-related transcription factor 2 (Runx-2) [47] has been investigated.

Bone homeostasis is maintained by osteoblasts (OBs) and osteoclasts (OCs) within the basic multicellular unit, in a consecutive cycle of resorption and formation. Therefore, a functional scaffold should allow the best possible OB/OC cooperation for bone remodeling, as happens within the bone extracellular matrix in the body [51]. As a current treatment, bone grafts are associated with inherent limitations; hence, bone tissue engineering as an alternative therapeutic approach has been considered in recent decades. Through the concerted participation and combination of biocompatible materials, osteoprogenitor/stem cells and bioactive factors closely mimic the bone microenvironment. Bioactive factors regulate the cell behavior, and they induce osteogenic differentiation of stem cells by activating specific signaling cascades [52].

Mesenchymal stem cells (MSCs) secrete different types of growth factors and cytokines, which, in turn, promotes tissue regeneration and attracts stem cell-like progenitor cells for recruitment and differentiation [53]. Therefore, MSCs are believed to be an important stem cell for tissue engineering, as well as being capable of differentiating into various specialized tissues, including bone, tendons, cartilage, muscle, ligaments, and fat $[54,55]$. The fabrication of macroporous PEG hydrogel scaffolds for use as a bioactive material for human bone marrow-derived MSCs in osteogenic differentiation has been successfully reported [56]. Another report also demonstrated that amphiphilic poly (d, 1-lactic acid)-co-poly (ethylene glycol)-co-poly (d, l-lactic acid) (PELA) with HA, fabricated by electrospinning, can lead to osteogenic differentiation of MSCs [57]. Furthermore, 3D MSC growth on PEG-HA biomimetic structures can enhance the polymerization process in the modulation of osteogenesis [58].

The purpose of this study was to establish a biomimetic nanostructured PEG-HAAuNP substrate for improving material properties by having more hydrophilicity, biocom- 
patibility, and anti-inflammatory capability, and establishing the osteogenic differentiation of MSCs, which were assessed through in vitro and in vivo assays.

\section{Materials and Methods}

\subsection{Material Preparation}

2.1.1. Preparation of Polyethylene Glycol (PEG) Stock Solution

PEG $500 \mu \mathrm{M}$ (Sigma-Aldrich, Burlington, MA, USA) was diluted 25 times to produce a final concentration of $20 \mu \mathrm{M}$. The material used in this experiment was prepared by applying the solution to a culture dish or 6-well, 24-well, or 96-well plates. After standing for $30 \mathrm{~min}$, the coating solution was sufficiently brought into contact with the surface of the culture plate. The excess solution was then removed, becoming the material of the composite film to be used in the upcoming experiments.

\subsubsection{Preparation of Polyethylene Glycol-Hydroxyapatite (HA)}

Hydrochloric acid was diluted to $2 \mathrm{~N}$; then, $5 \mathrm{~mL} 2 \mathrm{~N} \mathrm{HCl}$ was added to $0.1 \mathrm{~g} \mathrm{HA}$ and shaken to complete the dissolution. Afterwards, HA at a concentration of $20 \mathrm{mg} / \mathrm{mL}$ was diluted with PEG to obtain a PEG-HA solution $(2.5 \mathrm{mg} / \mathrm{mL})$. The mixing ratio was calculated from the mass conservation formula: M1V1 = M2V2 (M: concentration of solution; V: volume of solution). After coating for $30 \mathrm{~min}$, the solution was sufficiently in contact with the surface of the glass. The excess solution was then removed and neutralized with $1 \mathrm{~mL}$ of $0.05 \% \mathrm{NaOH}$ solution for $10 \mathrm{~min}$, prior to being washed with phosphate buffer solution (PBS).

\subsubsection{Preparation of Polyethylene Glycol-Hydroxyapatite-Gold Nanoparticles (PEG-HA-AuNPs)}

Gold nanoparticles (AuNPs) were purchased from Gold NanoTech Inc (Taipei, Taiwan). Gold NanoTech Inc utilizes unique and patented technology to physically break down gold into nanoparticles, followed by epitaxially stacking these nanoparticles into stacked materials with a controlled diameter within the nanometric range. Gold nanoparticles produced by this manufacturing process possess distinctive physical properties due to a special ionic charge that maintains their structure and are different from commercially available nanogold produced by chemical reduction methods. $\mathrm{HCl}$ was diluted to $2 \mathrm{~N}$; then, $5 \mathrm{~mL}$ of $2 \mathrm{~N} \mathrm{HCl}$ was added to $0.1 \mathrm{~g}$ of $\mathrm{HA}$ and shaken to the complete dissolution, at which time the HA concentration was $20 \mathrm{mg} / \mathrm{mL}$. HA was then diluted with PEG (20 $\mu \mathrm{M})$ to obtain an HA concentration in the PEG-HA solution of $2.5 \mathrm{mg} / \mathrm{mL}$. Then, an AuNP solution at a concentration of $1.25 \mathrm{ppm}$ was added to the PEG-HA solution, which was the working concentration used in the upcoming experiments. The mixing ratio was calculated from the mass conservation formula: M1V1 = M2V2 (M: concentration of solution; V: volume of solution). The material used in this experiment was prepared by applying the solution to a culture dish or 6-well, 24-well, or 96-well plates. After standing for $30 \mathrm{~min}$, the coating solution was sufficiently brought into contact with the surface of the culture plate. The excess solution was then removed, becoming the material of the composite film to later be used in the upcoming experiments.

\subsubsection{Preparation of Surface Coatings}

Different materials (PEG, HA, PEG-HA, PEG-HA-AuNPs, and PEG-AuNPs) were coated on the culture dish by applying the materials at the optimal concentration to cover the culture dish, plate, or $15 \mathrm{~mm}$ round coverslip glass. The coating solution was allowed to adsorb onto the surface of the culture area for 20-30 min. After coating, the residual content of the different materials was removed without any washing in order to establish a thin surface coating layer prior to cell culture. 


\subsection{Material Characterization}

\subsubsection{Fourier Transform Infrared Spectroscopy (FTIR) Analysis}

Fourier transform infrared spectrometry is derived from the Fourier transform, which requires the conversion of raw data into actual spectra. This experiment used potassium bromide (KBr) at $0.06 \mathrm{~g}$ (Sigma-Aldrich, Burlington, MA, USA), mixed with $200 \mathrm{~mm}$ of each experimental group, namely, PEG, PEG-HA, PEG-HA-AuNPs, and PEG-AuNPs. The scanning range was $600-4000 \mathrm{~cm}^{-1}$. A table change in surface functional groups was observed to demonstrate the different functional groups for each of the test materials [59].

\subsubsection{Surface-Enhanced Raman Scattering}

The Raman examination was processed on a Raman microscope (LABRAM HR UVVISNIR Version) system, following our previously published report [59]. Materials were placed on a silicon substrate, and detection of the holographic grating with CCD was calculated over a total time of $60 \mathrm{~s}$.

\subsubsection{UV-Visible Spectroscopy}

A spectrophotometer measures the range of waves from 190 to $1100 \mathrm{~nm}$, where the peak at $520 \mathrm{~nm}$ is the absorption wavelength of AuNPs. First, the quartz colorimetric tube must be cleaned with deionized water and wiped dry with mirror paper, and then the sample is added to the quartz colorimetric tube with deionized water for background absorption. After completion, each sample solution is measured sequentially. Prior to measuring the different samples, the quartz tube must be cleaned with deionized water to eliminate any residuals from the previous sample which could affect the absorption wavelength of the next sample. Origin Pro 8 (Originlab Corporation, Northampton, MA, USA) software analysis measured and quantified the data.

\subsubsection{Atomic Force Microscopy (AFM)}

The material surface morphology was observed through atomic force microscopy (MFP-3D, Asylum Research, Santa Barbara, CA), as reported in our previous work [59]. Initially, $100 \mu \mathrm{L}$ samples were cast on a Si (100) wafer substrate and then dried with blown air. Then, a silicon cantilever with a spring constant under $2.0 \mathrm{~N}$ constant conditions was used to observe the topography map of the materials. The resolution of the images processed in AC mode was $512 \times 512$ pixels. AFM results were obtained in three different experiments with samples at different scan areas.

\subsubsection{X-ray Photoelectron Spectroscopy (XPS)}

The elemental composition of the materials was assessed by X-ray photoelectron spectroscopy (XPS) (JEOL JPS 9010 MX, JEOL Ltd., Akishima, Tokyo, Japan). The radiation source was emitted from $\mathrm{Mg} \mathrm{K} \alpha$ X-rays [59].

\subsubsection{Free Radical Scavenging Ability}

2,2-diphenyl-1-picrylhydrazyl (DPPH, Sigma-Aldrich, Burlington, MA, USA) was used to evaluate the free radical scavenging ability of AuNPs and polymer composites [60]. A control of distilled water $(1 \mathrm{~mL})$ or $1 \mathrm{~mL}$ of deionized water containing PEG and a PEG composite was added to $3 \mathrm{~mL}$ of DPPH in methanol and then left to stand for $90 \mathrm{~min}$. The absorbance of the reaction mixture was then measured at $539 \mathrm{~nm}$ with an ultraviolet-visible spectrophotometer (Helios Zeta, Thermo, Waltham, MA, USA). The free radical scavenging ability effect is determined by the following equation: scavenging ratio $(\%)=[1-($ absorbance of test sample/absorbance of control $)] \times 100 \%$.

\subsection{Biocompatibility Test}

\subsubsection{Cell Culture Condition}

MC3T3-E1 Subclone 14 cells were obtained from ATCC. Cells were cultured in $\alpha$-MEM culture medium ( $10 \% \mathrm{FBS}, 100 \mathrm{U} / \mathrm{mL} \mathrm{P} / \mathrm{S}, 1 \%$ sodium pyruvate) and then removed with 
$0.05 \%$ trypsin-EDTA after reaching confluence at $37{ }^{\circ} \mathrm{C}, 5 \% \mathrm{CO}_{2}$. MSCs were isolated from Wharton's jelly tissue from the human umbilical cord [61]. Cells were cultured in a high-glucose DMEM culture medium (10\% FBS, $100 \mathrm{U} / \mathrm{mL} \mathrm{P} / \mathrm{S}, 1 \%$ sodium pyruvate) and then removed with $0.05 \%$ trypsin-EDTA after reaching confluence at $37{ }^{\circ} \mathrm{C}, 5 \% \mathrm{CO}_{2}$. For osteogenic differentiation, dexamethasone $(0.1 \mu \mathrm{M}$, Sigma, USA) and ascorbic acid-2phosphate (0.25 mM, Sigma, USA) were used.

For the characterization of MSCs, the specific surface markers of MSCs were characterized by flow cytometry. In brief, MSCs were detached, washed, and incubated with the indicated antibody conjugated with fluorescein isothiocyanate (FITC) and / or phycoerythrin (PE), against the indicated markers: CD14-FITC, CD34-FITC, CD44-PE, CD45-FITC, CD73-PE, and CD90-PE (BD Pharmingen, San Diego, CA, USA). PE-conjugated IgG1 and FITC-conjugated IgG1 were used as isotype controls (BD Pharmingen). Next, the antibody conjugated cells were analyzed by FACS analysis (LSR II, Becton Dickinson, Canton, MA, USA). MSCs in the 8th passage were used in this study.

\subsubsection{MTT Assay}

3-(4, 5-dimethylthiazol-2-yl)-2, 5-diphenyl tetrazolium bromide (MTT) was used as a substrate to react with mitochondrial dehydrogenase in living cells. Cells $\left(1 \times 10^{4} /\right.$ well $)$ were cultured on a 96-well plate coated with PEG, PEG-HA, PEG-HA-AuNPs, and PEGAuNPs after 24,48 , and $72 \mathrm{~h}$ of incubation. After incubation, cells were washed, and $100 \mu \mathrm{L}$ of MTT reagent $(0.5 \mathrm{mg} / \mathrm{mL})$ was added to each well at $37^{\circ} \mathrm{C}$ for $2-4 \mathrm{~h}$. Afterwards, $100 \mu \mathrm{L}$ of dimethylsulfoxide (DMSO) was added, and incubation was carried out for $10 \mathrm{~min}$. The absorbance was read by an ELISA reader at $570 \mathrm{~nm}$ (Molecular Devices, SpectraMax M2, Molecular Devices, San Jose, CA, USA) [44].

\subsubsection{Reactive Oxygen Species (ROS) Generation Analysis}

The substance test of the active peroxide was carried out using a fluorescent probe, DCFH-dA ( $2^{\prime}, 7^{\prime}$-dichlorofluorescin diacetate) (Sigma, USA). Cells $\left(2 \times 10^{5} /\right.$ well) were seeded in a 6-well plate coated with different materials at $37^{\circ} \mathrm{C}$ for $48 \mathrm{~h}$. After incubation, cells were collected, centrifuged, and washed with PBS. They were then stained with DCFH-dA $(10 \mathrm{nM})$ at $37^{\circ} \mathrm{C}$ for $30 \mathrm{~min}$ in dark conditions. The content of active oxidizing substances in the cells was calculated by a flow cytometer (BD, USA). Finally, ROS production was quantified using Flow Jo 7.6 (Becton Dickinson, Canton, MA, USA) software analysis [44].

\subsubsection{Actin Fiber Fluorescent Staining}

Cells $\left(1 \times 10^{4} /\right.$ well $)$ were seeded in a 24 -well plate coated with different materials for $8 \mathrm{~h}$ and $24 \mathrm{~h}$ of incubation. The cells were then fixed with $4 \%$ paraformaldehyde (PFA) for $10 \mathrm{~min}$ and permeated with $0.5 \%$ Triton X-100 in PBS, and then they reacted for $10 \mathrm{~min}$ at room temperature. Afterwards, phalloidin $(\sim 6 \mu \mathrm{M})$ (Sigma, USA) was treated at room temperature for $60 \mathrm{~min}$ in dark conditions. Finally, 4, 6-diamidion-2-phenylindole (DAPI, (Invitrogen, White Plains, NY, USA) dye was diluted at a concentration of $1 \mu \mathrm{g} / \mathrm{mL}$ and allowed to stain the nucleus in the dark for $10 \mathrm{~min}$ at room temperature. Completing the process, coverslips were sealed with Gel Mount ${ }^{\mathrm{TM}}$ (Sigma, USA), and the cytoskeletal patterns were observed under a fluorescent microscope [44].

\subsubsection{Cell Morphology and Adhesion Ability}

Scanning electron microscopy (JEOL JEM-5200, JEOL Ltd., Akishima, Tokyo, Japan) was applied to observe the morphology and attachment ability of cells grown on different materials. Briefly, cells $\left(1 \times 10^{4} / \mathrm{mL}\right)$ were cultured for $48 \mathrm{~h}$, fixed in a $2.5 \%$ glutaraldehyde solution for $8 \mathrm{~h}$, and then dehydrated with ethanol at a concentration of $30 \%$ to $100 \%$. Finally, after being dried at a critical point, the morphology of the cells on different materials was examined by SEM [44]. 


\subsubsection{Monocyte Activation Test}

Whole blood was obtained from an adult human. The blood sample was diluted with PBS (1:1 ratio), Ficoll was added to it, and then it was centrifuged at $2000 \mathrm{rpm}$ for $20 \mathrm{~min}$. Afterwards, the plasma layer was removed and the buffy coat was collected. Cells $\left(1 \times 10^{5} /\right.$ well $)$ were seeded in a 24 -well plate coated with different materials for $96 \mathrm{~h}$ of incubation in an RPMI condition medium (10\% FBS and 1\% $(v / v)$ antibiotics $(10,000 \mathrm{U} \mathrm{mL} /$ penicillin $\mathrm{G}$ and $10 \mathrm{mg} \mathrm{mL} /$ streptomycin) $)$. After incubation, cells were separated in the 24 -well plate using $0.05 \%$ trypsin. Finally, the ratio of monocytes and macrophages was observed under a microscope. To further confirm the inflammatory response, CD68 (as a marker of macrophages) was also confirmed by immunofluorescence staining as described in a previous report [44].

\subsubsection{Platelet Activation Test}

Platelets $\left(2 \times 10^{6}\right.$ platelets/well) were cultured on different materials for a $24 \mathrm{~h}$ incubation period, fixed with a $2.5 \%$ glutaraldehyde solution for $8 \mathrm{~h}$, and dehydrated with ethanol at a concentration of $30 \%$ to $100 \%$. After $8 \mathrm{~h}$, they were washed twice with PBS and dehydrated using a $30 \%$ to $100 \%$ alcohol concentration before the alcohol was removed after standing at room temperature for $10 \mathrm{~min}$ during each step. Finally, after being dried at a critical point, the morphology of the cells on different materials was examined by SEM (JEOL JEM-5200, USA), as described above [44].

\subsection{Biological Functional Assay}

\subsubsection{Cell Migration Assay}

The following cell migration assay procedure was implemented as reported in our previous published report [44]. Briefly, cells $\left(1 \times 10^{4} /\right.$ well $)$ were seeded in different materials through the use of a migration assay kit (Platypus Technologies, Madison, WI, USA) for a 96-well plate using a stopper. The stopper was removed, and incubation occurred for both $24 \mathrm{~h}$ and $48 \mathrm{~h}$. Afterwards, Calcein AM ( $2 \mu \mathrm{M})$ was applied and stained for 30 min at each time point, prior to the cell migration ability being observed under a fluorescence microscope. The extent of cell migration was analyzed by Image J (National Institutes of Health, Bethesda, MD, USA) software.

\subsubsection{Gelatin Zymography Analysis}

Cells $\left(2 \times 10^{5} /\right.$ well) were seeded into a 6-well plate overnight to allow the cells to attach. After $48 \mathrm{~h}$ of incubation, the culture medium was collected and determined as previously outlined. Samples were separated by $10 \%$ SDS-PAGE containing $2 \%$ gelatin, and the gel was incubated with a denaturing buffer $(40 \mathrm{mM}$ Tris- $\mathrm{HCl}, \mathrm{pH} 8.5,0.2 \mathrm{M} \mathrm{NaCl}, 10 \mathrm{mM}$ $\mathrm{CaCl}_{2}$, and $2.5 \%$ Triton $\mathrm{X}-100$ ) for $30 \mathrm{~min}$ at room temperature. The gel was then slowly stirred at room temperature and equilibrated with a development buffer $(40 \mathrm{mM}$ Tris- $\mathrm{HCl}$, $\mathrm{pH} 8.5,0.2 \mathrm{M} \mathrm{NaCl}, 10 \mathrm{mM} \mathrm{CaCl}_{2}$, and $0.01 \% \mathrm{NaN}_{3}$ ) for at least $16-18 \mathrm{~h}$ until it became activated in a $37{ }^{\circ} \mathrm{C}$ water bath. Afterwards, the gel was stained with $0.2 \%$ Coomassie Brilliant Blue R-250 (10\% acetic acid and 50\% methanol) and washed in a destaining buffer (10\% acetic acid, 20\% methanol). After Coomassie Blue staining, the protease-digested gelatin area appears as clear bands against a dark blue background. The resulting gel was digitized by scanning in a densitometer, and MMP gelatinase activity was quantified by Image Pro Plus 5.0 software (Media Cybernetics, Burlington, MA, USA).

\subsubsection{Enzyme-Linked Immunosorbent Assay}

Murine macrophage RAW 264.7 cells were kindly provided by Professor Hui-Jen Chen and cultured in high-glucose Dulbecco's modified Eagle's medium supplemented with $10 \%$ fetal bovine serum, $100 \mu \mathrm{g} / \mathrm{mL}$ streptomycin, and $100 \mathrm{U} / \mathrm{mL}$ penicillin at $37^{\circ} \mathrm{C}$ in $5 \% \mathrm{CO}_{2}$. The cells were passaged every 2 days, and cells were seeded at the density of $1 \times 10^{5}$ cells in each well (6-well plate). The cells were cultured in the different materials. After incubation for 12, 24, and $48 \mathrm{~h}$, the medium was collected and then centrifuged at 
$210 \mathrm{~g}$ to obtain supernatants for an ELISA assay. The concentrations of TNF- $\alpha$, IL- $1 \beta$, IL-6, IL-10, and VEGF were measured with ELISA kits (R\&D System) according to the manufacturer's instructions. The concentrations of these cytokines were analyzed based on standard curves, and the results are shown as the amount (pg) of TNF- $\alpha$ IL-1 $\beta$, IL-6, IL-10, and VEGF per ml of supernatant.

\subsubsection{Real-Time PCR Assay}

The total amount of RNA in the cells was extracted by Trizol (lnvitrogen, Waltham, MA, USA). The method provided in the manufacturer's manual was followed. Cells $\left(1 \times 10^{5} /\right.$ well) were seeded into a $10 \mathrm{~cm}$ culture dish after 7, 14, and 21 days of incubation. They were then treated with $1 \mathrm{~mL}$ of Trizol for $5 \mathrm{~min}$, RNA was extracted by adding $200 \mu \mathrm{L}$ chloroform (Sigma, USA) for $15 \mathrm{~s}$, and then they were kept for $3 \mathrm{~min}$ at room temperature prior to being centrifuged at $12,000 \mathrm{rpm}$ for $15 \mathrm{~min}$ at $4{ }^{\circ} \mathrm{C}$. The supernatant was removed and $500 \mu \mathrm{L}$ of isopropanol was added at $4^{\circ} \mathrm{C}$ for $10 \mathrm{~min}$. Finally, the samples were centrifuged at $12,000 \mathrm{rpm}$ for $15 \mathrm{~min}$ at $4{ }^{\circ} \mathrm{C}$. The supernatant was removed and washed twice with $1 \mathrm{~mL}$ of alcohol (75\%). After drying the RNA, $20 \mu \mathrm{L}$ of a DEPC-treated $\mathrm{H}_{2} \mathrm{O}$-soluble precipitate was added and quantified by reading the absorbance at $260 \mathrm{~nm}$ using an ELISA reader (Molecular Devices, SpectraMax M2, USA).

cDNA synthesis was performed using a RevertAidTM First Strand cDNA DNA Synthesis Kit (Fermentas, Canada) following the manufacturer's procedures. First, $2 \mu \mathrm{L}$ of oligo (dT) 18 primer and random hexamers (1:1) were added to the RNA sample and placed in a gradient polymerase reaction temperature controller at $65{ }^{\circ} \mathrm{C}$ for $5 \mathrm{~min}$. Then, the addition of $4 \mu \mathrm{L}$ of $5 \times$ reaction buffer, $1 \mu \mathrm{L}$ LockTM RNase inhibitor $(20 \mathrm{U} / \mathrm{mL}), 2 \mu \mathrm{L}$ dNTP Mix (10 mM), and $1 \mu \mathrm{L}$ of RevertAidTM M-MuLV Reverse Transcriptase $(200 \mathrm{U} / \mathrm{mL})$ proceeded, before being reacted at $42{ }^{\circ} \mathrm{C}$ for $60 \mathrm{~min}$. Finally, each sample underwent a reaction at $70{ }^{\circ} \mathrm{C}$ for $5 \mathrm{~min}$ to obtain cDNA.

The polymerase chain reaction was carried out using the cDNA as a template and a 1Q2 Fast qPCR System with a reaction volume of $10 \mu \mathrm{L}$ according to the manufacturer's procedures. Firstly, $0.5 \mu \mathrm{L}$ of primer $(0.3 \mu \mathrm{M})$ and $5 \mu \mathrm{L}$ of enzyme were added to the cDNA sample, with the RNA expression then analyzed using the Step OneTM Plus Real-Time PCR System.

\subsubsection{Alizarin Red S Staining (ARS)}

Cells $\left(1 \times 10^{5} /\right.$ well $)$ were seeded into a $10 \mathrm{~cm}$ culture dish after 7,14 , and 21 days of incubation. After incubation, the cells were fixed with $4 \%$ PFA for $15 \mathrm{~min}$ and washed with PBS. A 2\% Alizarin Red S staining solution was prepared and filtered, and the $\mathrm{pH}$ was adjusted to 4.1 4.3 so an Alizarin Red S working solution was made available. The cells were immersed in $500 \mu \mathrm{L}$ of $\mathrm{ddH}_{2} \mathrm{O}$ for $1 \mathrm{~min}$ and then drained and removed. A $500 \mu \mathrm{L}$ Alizarin Red S working solution was added after being reacted for $15 \mathrm{~min}$ at room temperature. Finally, it was drained and then soaked in $500 \mu \mathrm{L}$ of deionized water for $2 \mathrm{~min}$. The staining results were observed under a microscope and recorded.

\subsection{Rat Subcutaneous Implantation}

Sprague Dawley rats (300 350 g) were incised dorsally in an area of $10 \mathrm{~mm}^{2}$ under anesthesia prior to being subcutaneously implanted with the different materials. After 1 month of implantation, the samples were removed and examined using hematoxylin and eosin (H\&E). We calculated the thickness of the fibrous capsule over 6 sites using Image J software to quantify the average encapsulated fibrotic tissues. The monoclonal anti-CD86, anti-CD163, and anti-CD45 antibodies (1:200 dilution) (Santa Cruz, CA, USA) were used to evaluate the activation levels of M1 and M2 macrophages. The secondary antibodies AF488 donkey anti-mouse IgG (1:500 dilutions) (Invitrogen, Carlsbad, CA, USA) and anti-mouse Immunoglobulin G (rhodamine) (1:500 dilution) (Jackson Immuno Research, West Grove, PA, USA) were used for signal detection. An Olympus ix71 fluorescence microscope (Tokyo, Japan) was then equipped to measure the fluorescence intensity. The 
amount of collagen deposition was observed as blue color using a Masson trichrome staining kit (Sigma, USA) according to the manufacturer's instructions. The area of fibrosis tissue in the sample sections was calculated using Image J 4.5 version software (Media Gybertics). Three selected sections from randomly selected high-power fields (HPFs) were quantified for each animal and analyzed. After the number of pixels in each fibrosis area per HPF was measured, the number of pixels obtained from the three HPFs was collected and summed. The TUNEL assay is a procedure to detect DNA fragmentation by labeled $3^{\prime}$-hydroxyl termini in double-stranded DNA breaks which is generated during apoptosis. Paraffin-mounted heart sections from control and treated groups were deparaffinized with xylene and descending concentrations of ethanol. Apoptotic cells were stained based on the protocol provided by the In Situ Cell Death Detection Kit, AP (Roche Diagnostics, Indianapolis, IN, USA). DAPI solution was used to stain nuclei for investigation by a confocal microscope [62]. The number of rats was $5(n=5)$. Results are mean \pm SD. All procedures followed the ethical guidelines and were approved by the animal care and use committee (La-1071565).

\subsection{Statistical Analysis}

Experiments were independently performed in triplicate to avoid uncertainty. Data for each test $(n=3-6)$ were collected and are presented as mean \pm standard deviation. Student's $t$-test and the single-factor analysis of variance (ANOVA) method were used to examine the differences between the groups. For ANOVA, Bonferroni was chosen for post hoc analysis. A $p$ value less than 0.05 was considered to be statistically significant.

\section{Results}

The material preparation and surface morphology identification are illustrated in Figure 1A. The assembly mechanism of PEG-HA-AuNP nanocomposites may be attributed to the strong interaction between gold and oxygen atoms. This phenomenon implies that the additive interacts with the oxygen atoms in the PEG segment, causing the carbon atoms in the segment to be exposed to the surface. Indeed, it is also shown that the water contact angles of PEG, HA, PEG-HA, PEG-HA-AuNPs, and PEG-AuNPs were $24.9 \pm 0.05^{\circ}$, $37.5 \pm 0.45^{\circ}, 17.8 \pm 0.19^{\circ}, 15.3 \pm 0.46^{\circ}$, and $15.0 \pm 0.22^{\circ}$, respectively (Figure $1 \mathrm{~B}$ ). The hydrophilicity property of materials is critical for the attachment of cells to the extracellular matrix (ECM) through cell adhesion molecules. In the present study, the addition of AuNPs to PEG-HA and PEG made the polymer surface more hydrophilic (Figure 1B), suggesting that the nanocomposites could facilitate the adhesion ability of MC3T3 cells and MSCs. These signals were clearly observed in PEG-HA and PEG-HA-AuNPs. UV-Vis spectroscopy was used to measure the absorption wavelength of the gold nanoparticles (AuNPs). The pure AuNPs have a typical peak at $520 \mathrm{~nm}$ and were observed in the PEGHA-AuNP and PEG-AuNP groups (Figure 1C). As shown in Figure 1D, the specific peaks of PEG are $2931 \mathrm{~cm}^{-1} v\left(-\mathrm{CH}_{2}\right)_{\text {asym }}, 2868 \mathrm{~cm}^{-1}\left(\mathrm{CH}_{3}\right)$ sym, and $1105 \mathrm{~cm}^{-1} v(\mathrm{OH})$, with these three peaks being found in the PEG-HA, PEG-HA-AuNP, and PEG-AuNP groups, respectively, thus demonstrating the presence of HA and AuNPs in PEG. However, FTIR can only be used for the detection of organic functional groups and not for inorganic functional groups. Therefore, Raman spectroscopy was used to further confirm this result. As shown in Figure 1E, the specific peaks of polyethylene glycol are $1080 \mathrm{C}-\mathrm{C}, v\left(\mathrm{CH}_{2}\right)$ $\mathrm{cm}^{-1}, 1141 \mathrm{C}-\mathrm{C}, v\left(\mathrm{CH}_{2}\right) \mathrm{cm}^{-1}, 1255 \mathrm{~cm}^{-1} v\left(\mathrm{CH}_{2}\right) \mathrm{cm}^{-1}, 1297 v\left(\mathrm{CH}_{2}\right) \mathrm{cm}^{-1}, 1482 \mathrm{~cm}^{-1} v$ $\left(\mathrm{CH}_{2}\right)_{\text {sym }} \mathrm{cm}^{-1}, 2892 \mathrm{~cm}^{-1}, v\left(\mathrm{CH}_{2}-\mathrm{CH}_{3}\right)_{\text {sym }} \mathrm{cm}^{-1}$, and $2930 v\left(\mathrm{CH}_{2}-\mathrm{CH}_{3}\right)_{\text {asym }} \mathrm{cm}^{-1}$. The characteristic peaks of hydroxyapatite are $904 \mathrm{~cm}^{-1}\left(\mathrm{C}_{2}-\mathrm{H}_{\text {deformation }}\right)$ and $1664 \mathrm{~cm}^{-1} \mathrm{C}=\mathrm{C}$ amide I. Indeed, the free radical scavenging ability of PEG and PEG nanocomposites is demonstrated in Figure 1F. The capture capacity of PEG-HA was significantly higher than that of PEG or PEG-Au nanocomposites $(p<0.05)$. It is mainly the exposed HA on the surface that allows PEG-HA to combine with more free radicals. On the other hand, Au nanoparticles aggregated in the PEG polymeric chain, which led to a decrease in the free radical scavenging ability. 

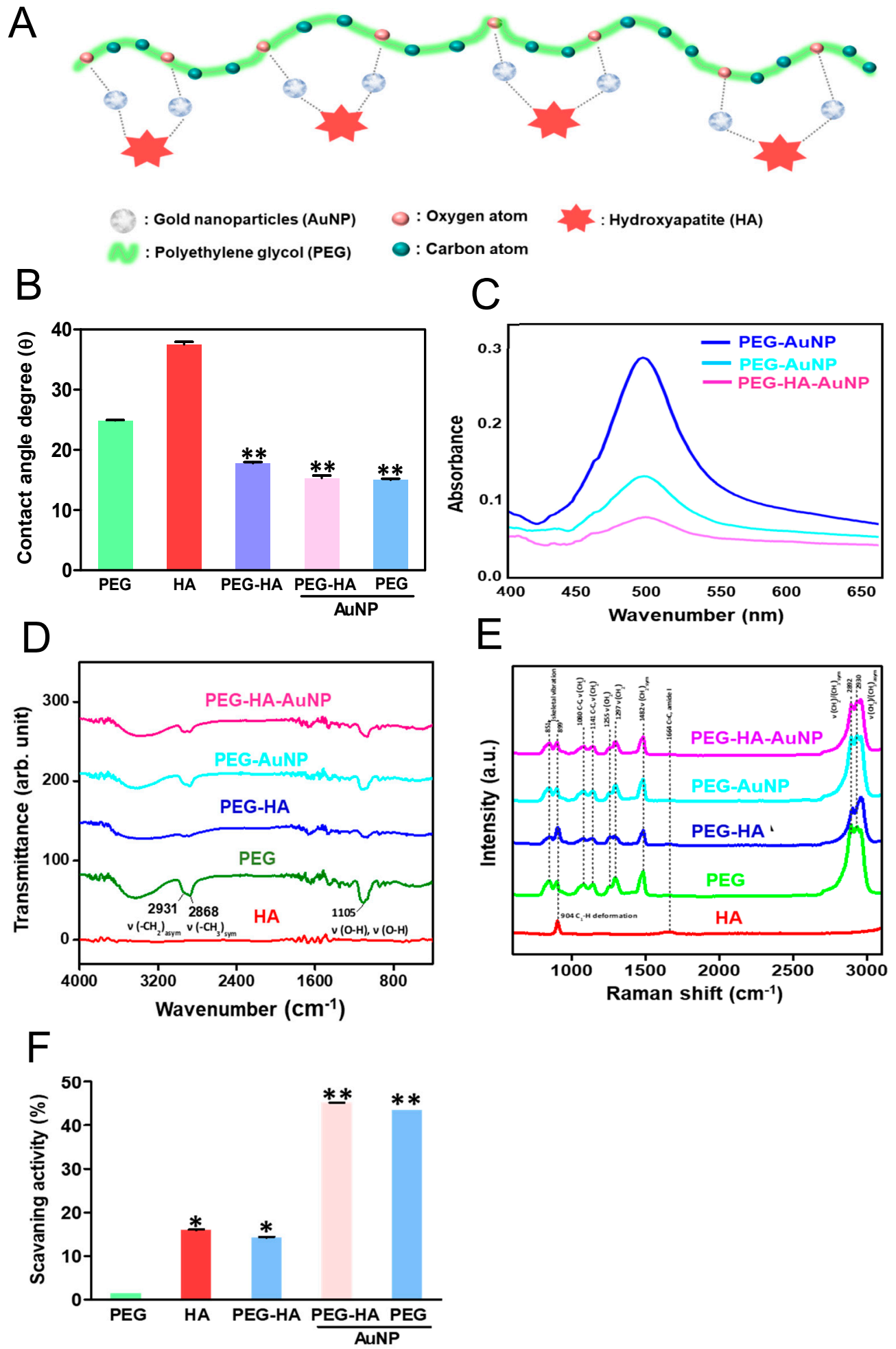

Figure 1. (A) Schematic diagram illustrating the preparation procedure of PEG-HA-AuNPs. Characterization of PEG-HAAuNP nanocomposites: (B) Average contact angle $(\theta)$ quantified from different materials. The contact angle from different materials without water is $\theta=0^{\circ}$. Data are the mean $\pm \operatorname{SD}(n=3){ }^{* *} p<0.01$, smaller than the control treatment (PEG). (C) UV-Vis spectra, (D) FTIR spectra, and (E) Raman spectra of different materials. (F) Free radical scavenging effect of HA, PEG, and PEG nanocomposites. Free radical scavenging effect of HA, PEG, and PEG nanocomposites. Mean \pm SD. ${ }^{*} p<0.05$; ${ }^{* *} p<0.01$ : greater than control.

The surface morphology of pure PEG had a uniform and homogenous property, while the pure HA showed an irregular strip shape with rough protrusions. However, when 
PEG was mixed with the HA, it was observed that the surface morphology of the material became smoother and more homogenous. In addition, when PEG-HA was crosslinked with AuNPs, it prominently revealed that the surface shape of PEG-HA had generated bulk laminar-like protrusions with a homogeneous property. Specifically, when the AuNPs were added to the pure PEG matrix, the surface form was converted into a smaller striplike nanostructure morphology (Figure 2). The surface roughness of PEG, HA, PEG-HA, PEG-HA-AuNPs, and PEG-AuNPs was $0.31 \mathrm{~nm}, 5.4 \mathrm{~nm}, 0.2 \mathrm{~nm}, 0.5 \mathrm{~nm}$, and $0.97 \mathrm{~nm}$, respectively (Figure 2A). This indicates that both PEG-HA-AuNPs and PEG-AuNPs had lower surface roughness conformity. Young's modulus was used to analyze the material stiffness for this assay. The results for Young's modulus show that PEG, HA, PEG-HA, PEG-HA-AuNPs, and PEG-AuNPs had Young moduli of $12 \mathrm{MPa}, 550 \mathrm{MPa}, 240 \mathrm{MPa}$, $130 \mathrm{MPa}$, and $6 \mathrm{MPa}$, respectively (Figure $2 \mathrm{~B}$ ). This shows that adding PEG and AuNPs into the HA matrix can cause the surface to become softer, particularly in the PEG-AuNP group. The stiffness of the material was investigated by measuring Young's modulus in this study. The addition of gold nanoparticles to the PEG matrix did not have a significant effect on its stiffness upon statistical analysis.

In addition, it was determined that when the incorporation of AuNPs into PEGHA occurred, the surface morphology of PEG-HA-AuNPs was significantly changed according to SEM analysis (Figure 3A). This result is similar to the surface pattern of the AFM image (Figure 2A). Therefore, both PEG-HA-AuNPs and PEG-AuNPs have better hydrophilicity properties, which is beneficial for cell attachment. It was then confirmed that surface fabrication of PEG-HA by AuNPs can effectively induce pure PEG-HA substrates to generate different surface morphological changes. The XPS spectra of PEG and PEG composites are shown in Figure 3B. The atomic concentrations of O, C, Ca, P, and Au were calculated using XPS area measurement. The O1s/C1s ratio was 1.77 (Table S1). When PEG contains gold nanoparticles or hydroxyapatite, the $\mathrm{O} 1 \mathrm{~s} / \mathrm{C} 1 \mathrm{~s}$ ratio of the sample surface gradually decreases. However, only faint signals of gold nanoparticles were observed by XPS. This may be due to the strong interaction between gold and oxygen atoms. This phenomenon implies that the additive interacts with the oxygen atoms in the PEG segment, causing the carbon atoms in the segment to be exposed to thesurface. 
A
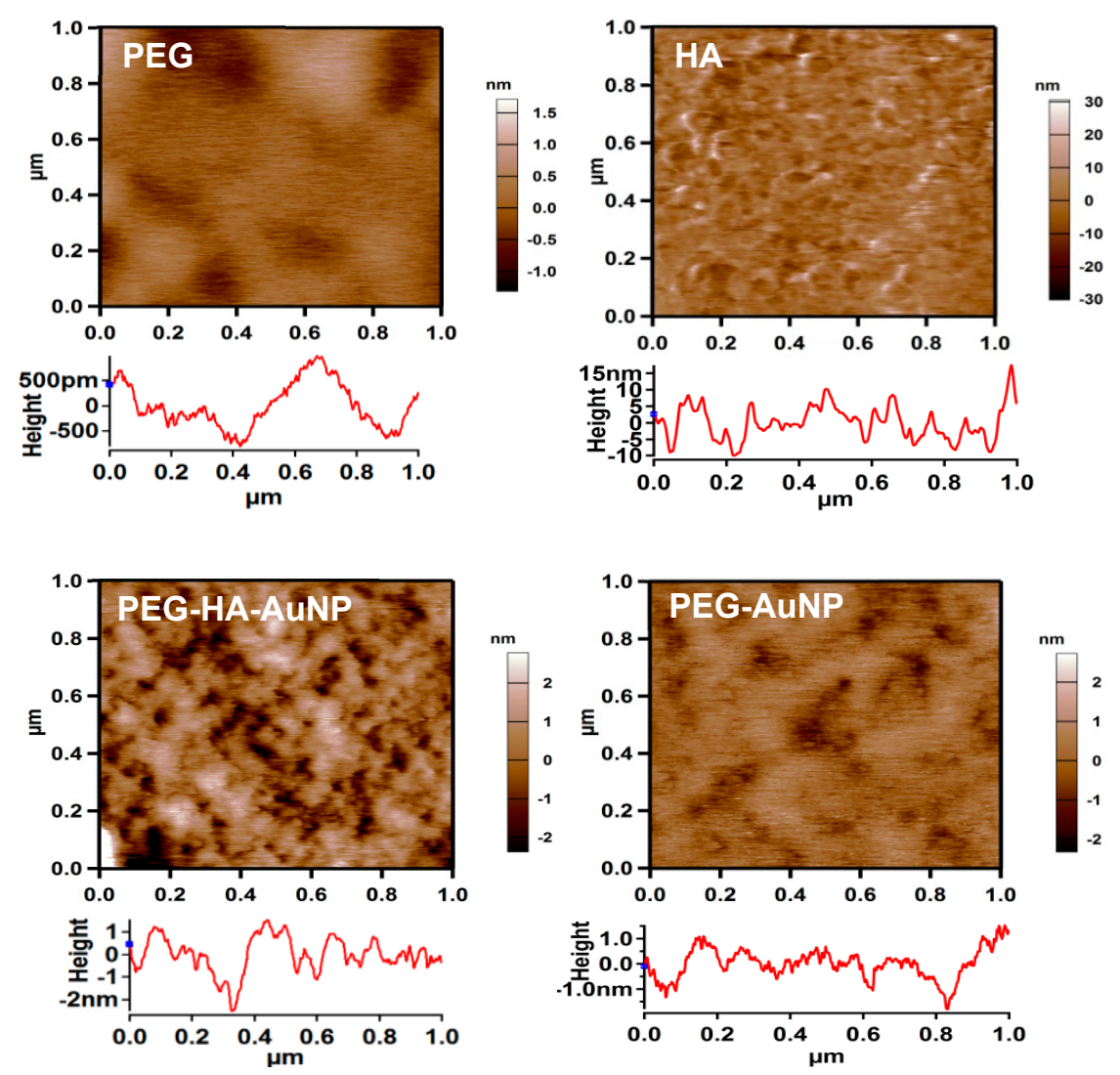

B
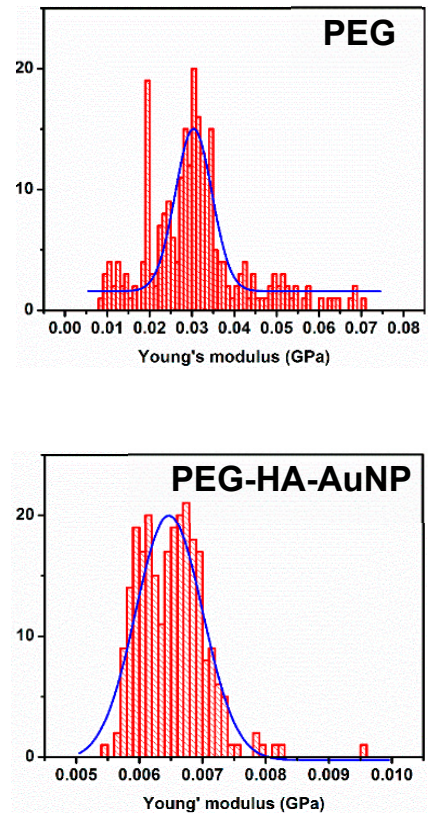
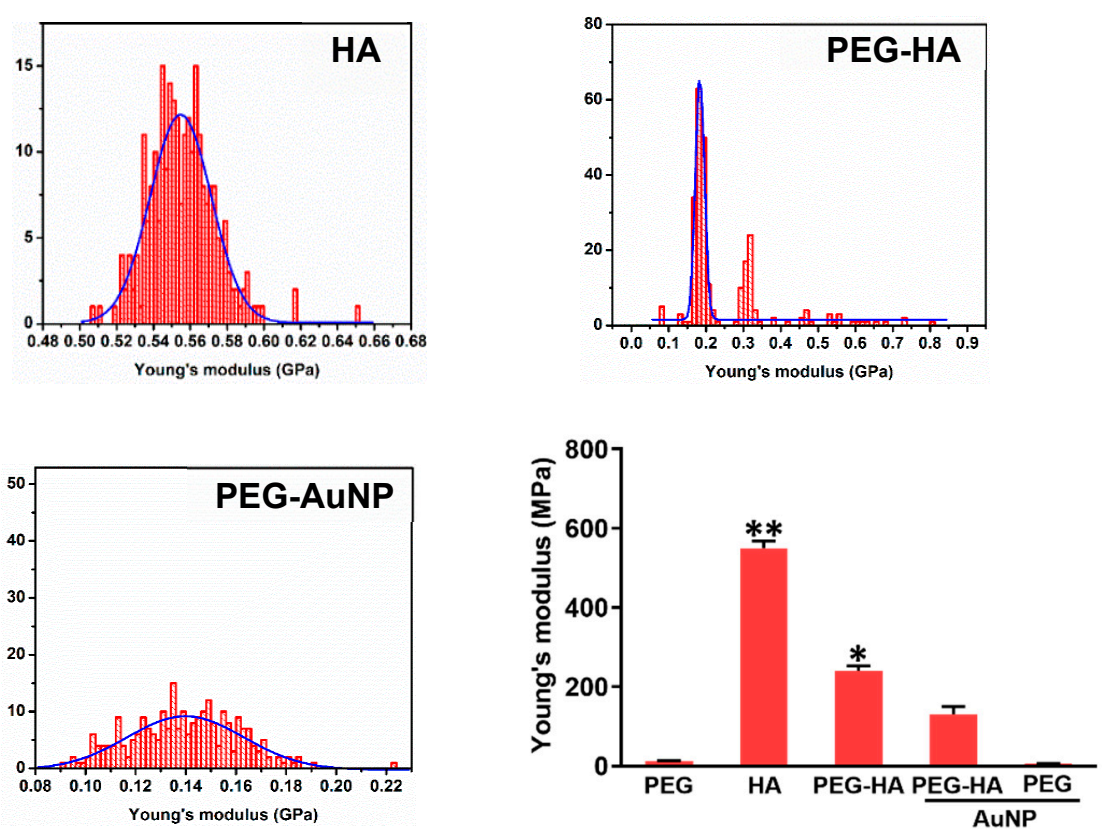

Figure 2. Surface roughness property characterization of different materials by AFM analysis. AFM images of different materials. (A) Topographical images of different materials. (B) Histograms of Young's modulus values (MPa) of different materials. The quantification data of surface roughness of AFM, and the quantification of Young's modulus of different materials. ${ }^{*} p<0.05 ;{ }^{* *} p<0.01$ : smaller than control. 
A
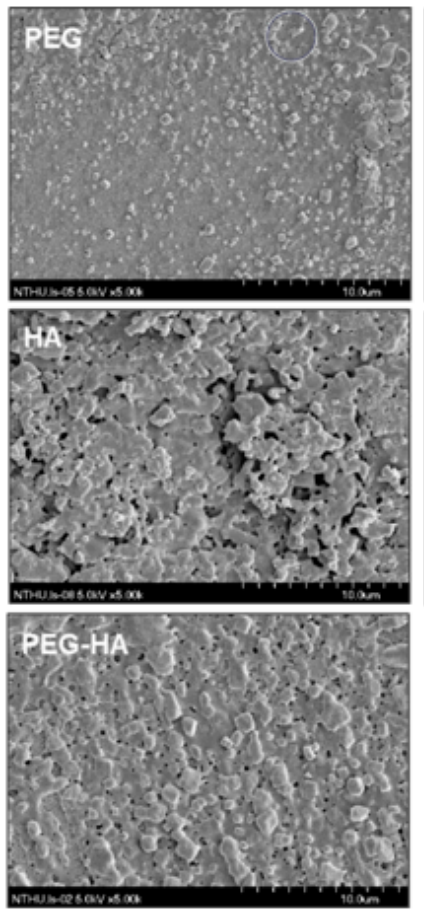

B
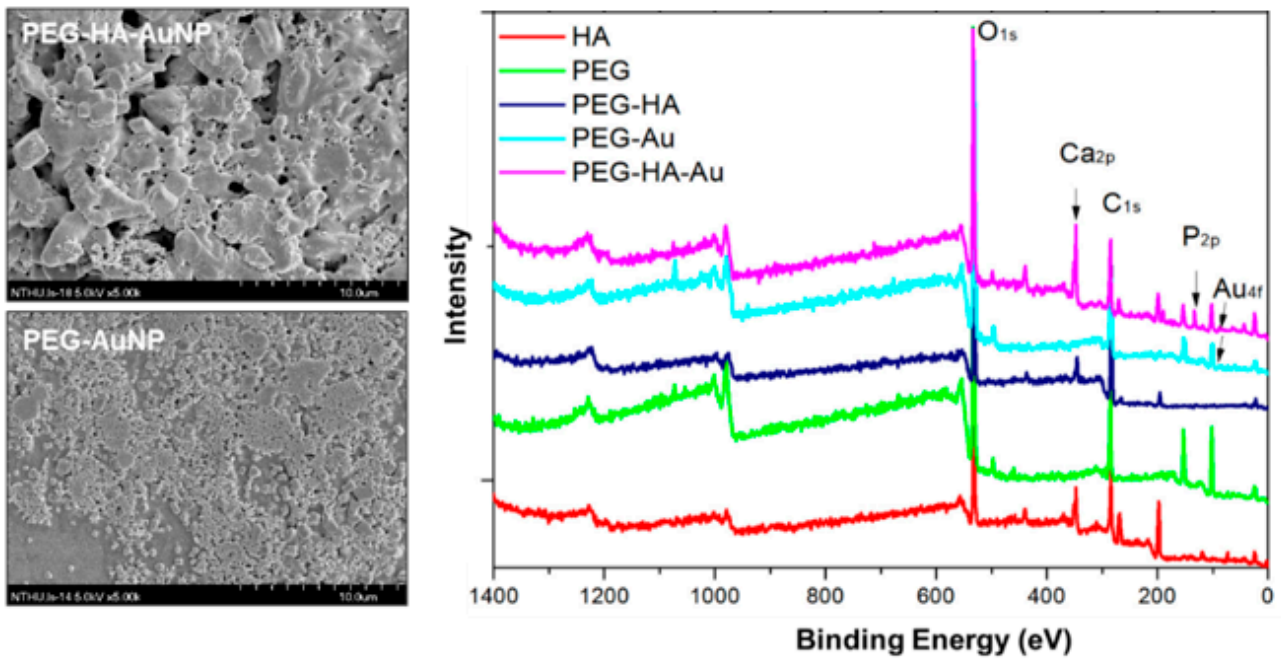

Figure 3. Material characterization. (A) SEM analysis of different materials. (B) Wide-scan spectra of PEG and PEG composites by XPS analysis.

The cell growth of MSCs and MC3T3 cells on PEG-HA-AuNPs after incubation for $48 \mathrm{~h}$ is shown in Figure $4 \mathrm{~A}$. The proliferation ability of MSCs at $48 \mathrm{~h}$ was the greatest on PEG-HA-AuNPs at $43.5 \mathrm{ppm}$, followed by $17.4 \mathrm{ppm}$ and $174 \mathrm{ppm}$. Furthermore, ROS generation was the lowest in the concentration of PEG-HA-AuNPs at $43.5 \mathrm{ppm}$, followed by $17.4 \mathrm{ppm}$ and $174 \mathrm{ppm}$, both in the MSCs and MC3T3 cells (Figure 4B). In addition, the CD68 fluorescence intensity on PEG-HA-AuNPs at $43.5 \mathrm{ppm}$ was smaller than that on the other materials (Figure 4C). Semi-quantification data were established through CD68 immunofluorescence staining $(p<0.01)$ (Figure 4E). Moreover, platelets from the control group and control (glass) were almost flattened (activated form) (Figure 4D). In contrast, platelets from PEG platelets were less activated on all PEG-HA-AuNP nanocomposites, particularly at the concentration of $43.5 \mathrm{ppm}$, followed by $17.4 \mathrm{ppm}$ and $174 \mathrm{ppm}(p<0.01)$ (Figure 4F). 

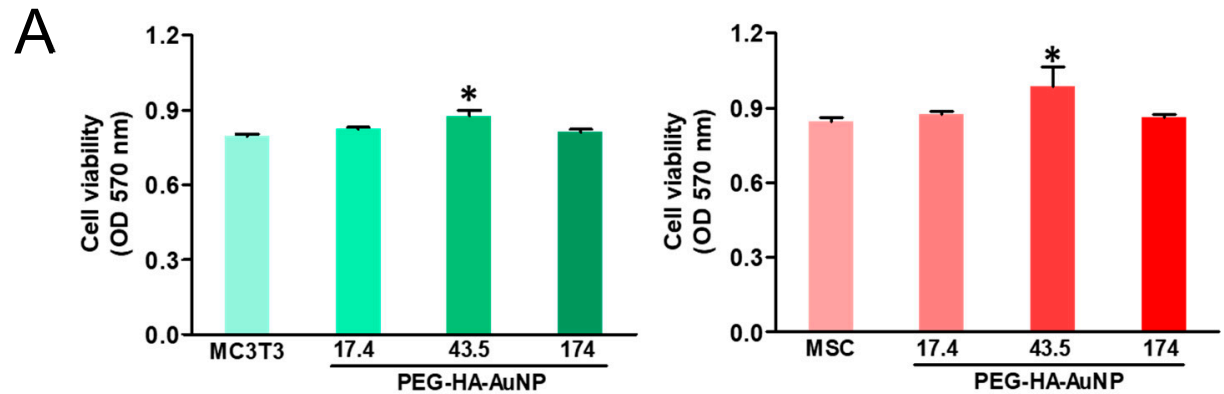

B
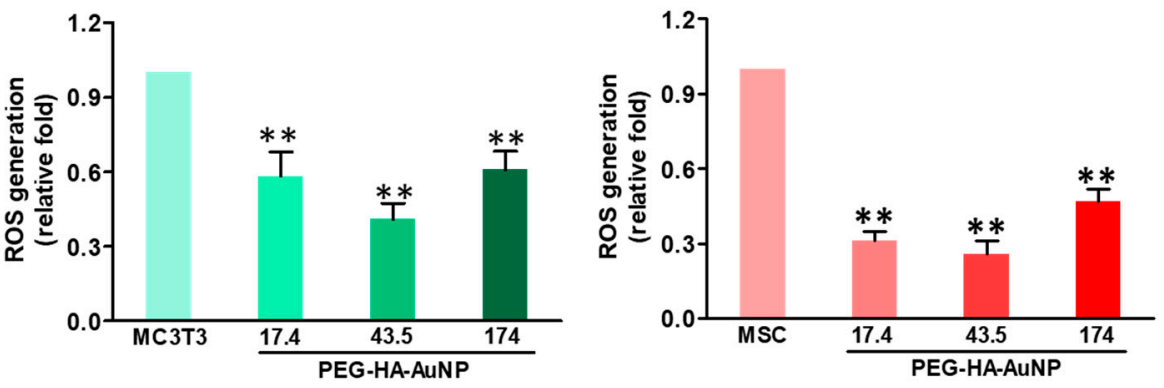

C
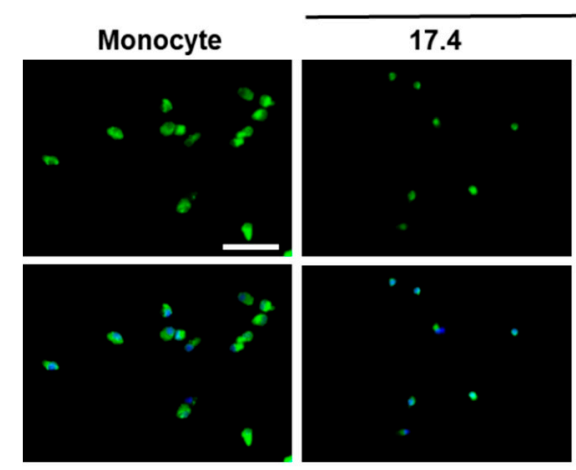

PEG-HA-AUNP
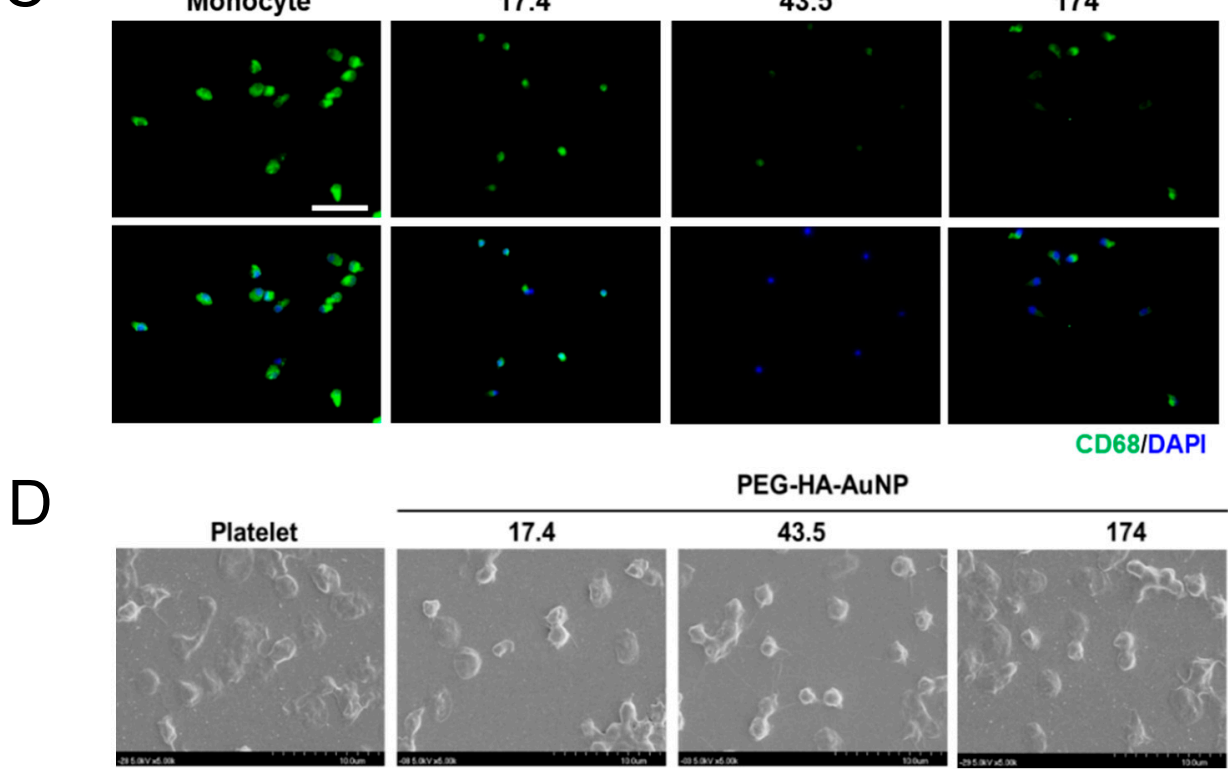

PEG-HA-AuNP

CD68/DAPI
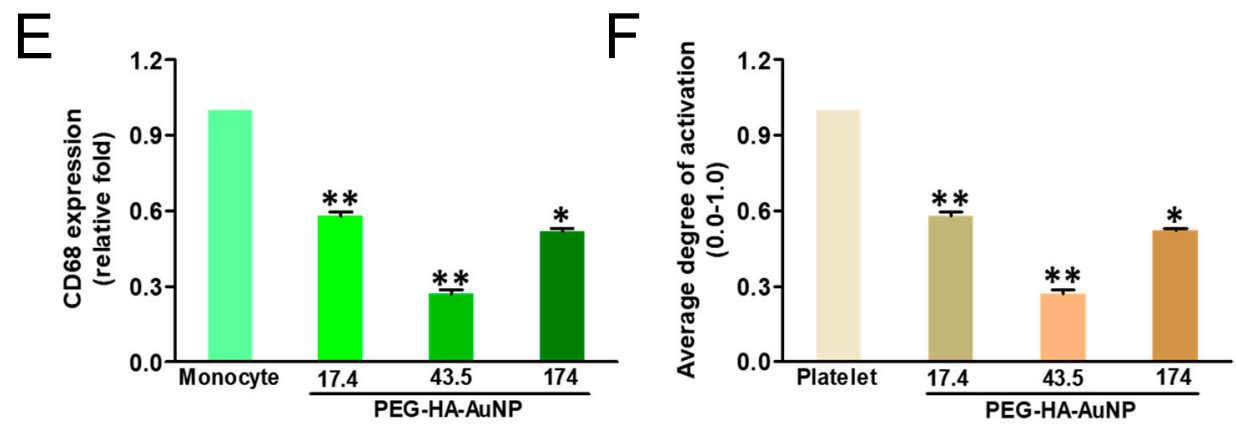

Figure 4. Cell proliferation of (A) MC3T3 cells and MSCs was promoted by culture on TCPS and different concentrations of AuNPs in the PEG-HA matrix. Data are the mean $\pm \mathrm{SD}(n=3),{ }^{*} p<0.05 ;{ }^{* *} p<0.05$. Reactive oxygen species (ROS) generation assay of (B) MC3T3 cells and MSCs on different materials after $48 \mathrm{~h}$ of incubation. Intracellular ROS quantified by 2,7-dichlorofluorescein diacetate (DCFH-dA) and flow cytometric analysis. ${ }^{*} p<0.05$; ${ }^{* *} p<0.01$ : smaller than control (TCPS). Biocompatibility assay. (C) The expression of CD68 for macrophages on different materials at 96 h. Cells were immunostained by the primary anti-CD68 antibody and conjugated with FITC-immunoglobulin secondary antibody (green 
color fluorescence). Cell nuclei were stained by DAPI (blue color fluorescence). Scale bar $=20 \mu \mathrm{m}$. (D) SEM images showing the adhesion and activation of human blood platelets on different materials. (E) CD68 expression was quantified based on fluorescence intensity. ${ }^{*} p<0.05$ : smaller than control (TCPS). (F) Quantification of the degree of platelet activation score. Data are the mean $\pm \mathrm{SD}(n=3)$. ${ }^{*} p<0.05 ;{ }^{* *} p<0.01$ : smaller than control (TCPS). Based on these findings, we thus chose PEG-HA-AuNPs at $43.5 \mathrm{ppm}$ in the following experiments.

We also characterized the MSC phenotypes by the detected surface markers of MSCs using FACS analysis (Figure S1A). We detected negative surface makers such as CD14, CD34, and CD45, which were expressed in hematopoietic cells, endothelial cells, and immune cells, respectively, and positive surface antigens of MSCs: CD44, CD73, and CD90. Data from the FACS analysis further show that less than $2 \%$ of the negative markers (Figure S1B) and higher than $98 \%$ of the positive markers were quantified (Figure S1C) and validated in MSCs.

The MTT results show that cell growth ability prominently increased in both PEGHA-AuNPs and PEG-AuNPs when compared to the control group, particularly after $72 \mathrm{~h}$ in MC3T3 cells $(p<0.01)$. This stronger growth pattern was also observed in MSCs while being cultured on the different materials after 24,48 , and $72 \mathrm{~h}$ of incubation. This was seen specifically in the PEG-HA-AuNP and PEG-AuNP groups, followed by the PEGAuNP, PEG-HA, and PEG groups, when compared to the control group (TCPS) $(p<0.01)$ (Figure 5A). Indeed, cells were cultured on different materials in order to investigate their intracellular ROS generation ability. While MC3T3 cell growth on different materials occurred over $48 \mathrm{~h}$, the average amount of ROS production of PEG-HA-AuNPs ( 0.69-fold) and PEG-AuNPs ( 0.67-fold) in the cells was lower than that of PEG ( 0.9-fold) and PEGHA ( 0.83-fold) when compared to the control group $(p<0.01)$ (Figure 5B). Indeed, a similar result occurred for MC3T3 cells while being cultured on different materials, as the anti-ROS generation ability was also observed after $24 \mathrm{~h}$ of incubation, specifically for the PEG-HA-AuNP and PEG-AuNP groups $(p<0.01)$ (Figure 5B). Similar to MC3TC cells, during MSC growth on different materials over $48 \mathrm{~h}$, the ROS generation ability of both PEG-HA-AuNPs ( 0.53-fold) and PEG-AuNPs ( 0.47-fold) was lower than that of PEG ( 0.9-fold) and PEG-HA ( 0.89-fold) when compared to the control group (TCPS) $(p<0.01)$ after $48 \mathrm{~h}$ of incubation. Indeed, the average amount of ROS produced in the MSCs was lower in the PEG-HA-AuNP and PEG-AuNP groups than the other groups after $24 \mathrm{~h}$ of incubation $(p<0.01)$ (Figure 5B). Based on these results, it can be suggested that the PEG-HA-AuNP and PEG-AuNP groups had better antioxidant abilities, as well as good biocompatibility, in both MC3T3 cells and MSCs. 

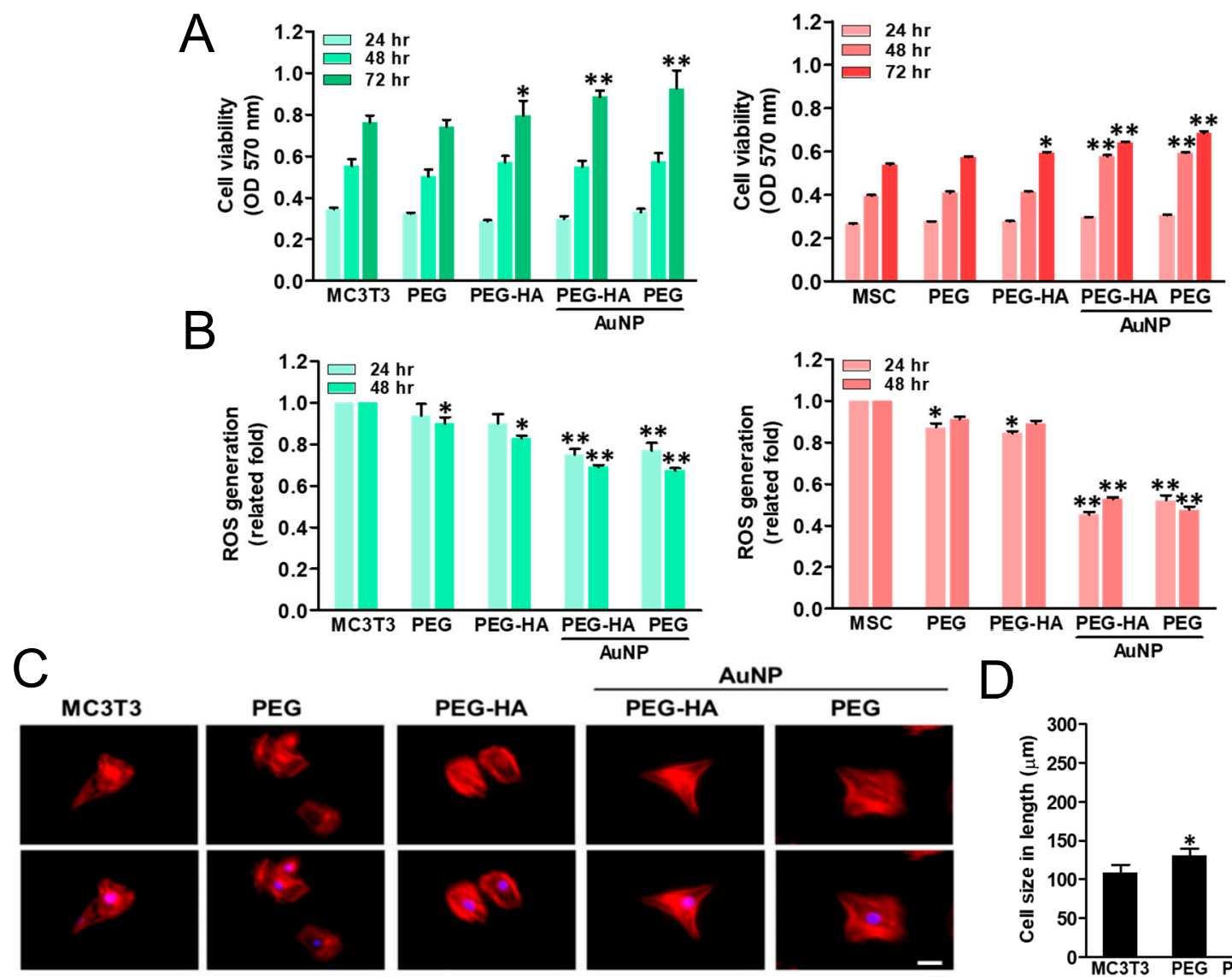

D
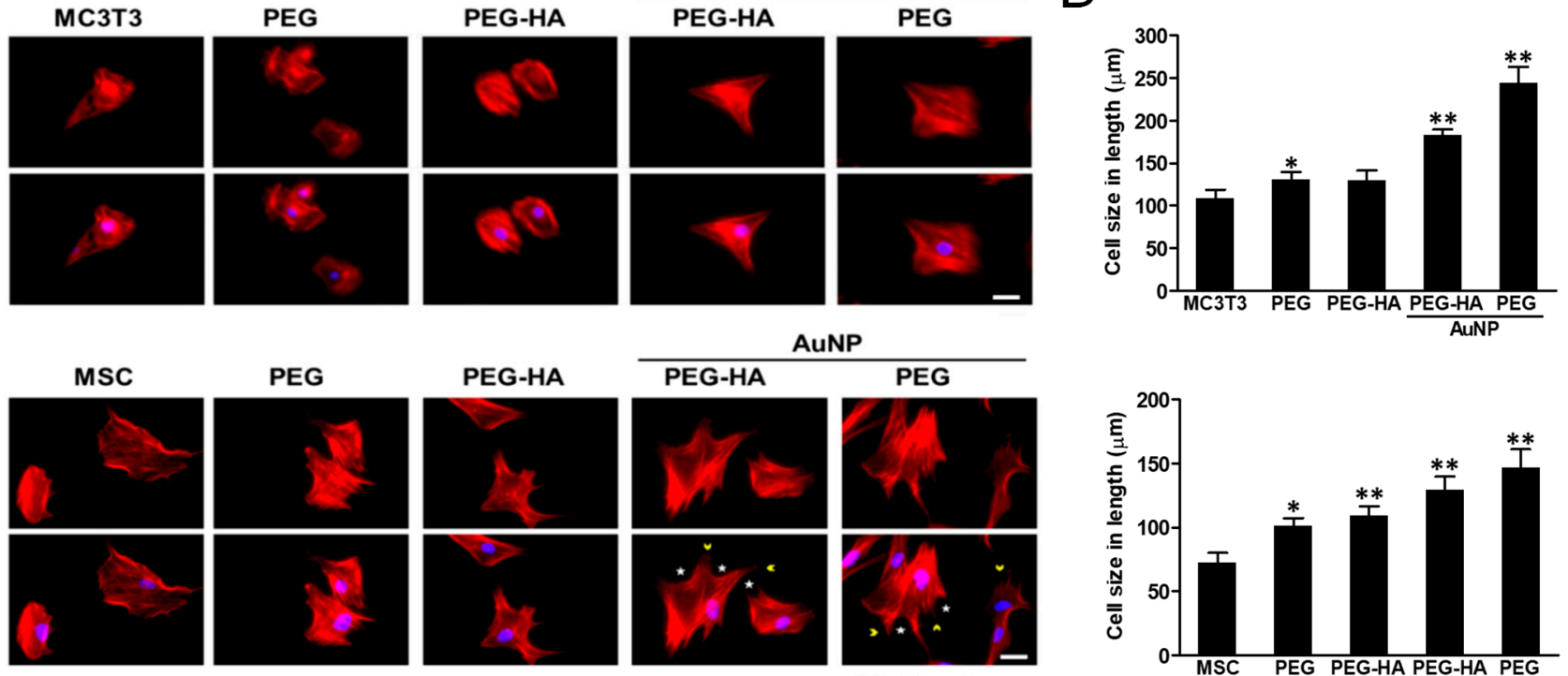

:Filopodium

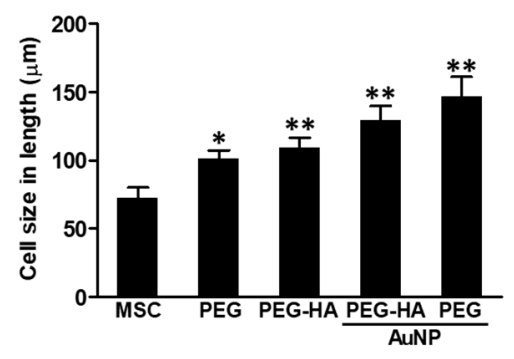

Figure 5. Cell proliferation of (A) MC3T3 cells and MSCs was promoted by culturing them on PEG-HA-AuNPs and PEGAuNPs. Data are the mean $\pm \mathrm{SD}(n=3),{ }^{*} p<0.05 ;{ }^{* *} p<0.05$. Reactive oxygen species (ROS) generation assay of (B) MC3T3 cells and MSCs on different materials after $48 \mathrm{~h}$ of incubation. Intracellular ROS quantified by 2,7-dichlorofluorescein diacetate (DCFH-dA) and flow cytometric analysis. ${ }^{*} p<0.05 ;{ }^{* *} p<0.01$ : smaller than control (TCPS). Cytoskeleton and cell morphology by rhodamine phalloidin staining of (C) MC3T3 cells and MSCs for actin fiber extension on different materials after $24 \mathrm{~h}$ of incubation under fluorescence microscopy analysis. Scale bar $=20 \mu \mathrm{m}$. Arrows indicate filopodia (green color) and lamellipodia (red color). Data are the mean $\pm \mathrm{SD}(n=3)$. Actin fiber extension in length quantified by Image J software in (D) MC3T3 cells and MSCs on different materials after $8 \mathrm{~h}$ is shown. Actin fiber length elongation was significantly observed in the PEG-HA-AuNP and PEG-AuNP test groups compared with the other groups. Data are mean $\pm \operatorname{SD}(n=3)$. ${ }^{*} p<0.05 ;{ }^{* *} p<0.05$. Scale bar $=50 \mu \mathrm{m}$.

The actin fiber extension of MC3T3 cells and MSCs on different materials was observed through phalloidin staining. In one control group (glass), the cell shape was circular. However, the cytoskeleton was spread out to stress the fiber, particularly for the PEG-HAAuNP and PEG-AuNP groups, followed by PEG-HA and PEG, in both MC3T3 cells and MSCs after $24 \mathrm{~h}$ of incubation (Figure 5C). At $24 \mathrm{~h}$ of incubation, the cell size of MC3T3 cells significantly increased in the control group (glass) (Figure 5C). Moreover, a similar 
pattern was also observed in MSCs. When MSCs attach or migrate on materials, they mainly produce lamellipodia and filopodia to change the morphology of cells (Figure 5C). Indeed, after $24 \mathrm{~h}$ of incubation, the cell sizes of MSCs in the control group, PEG, PEGHA, PEG-HA-AuNPs, and PEG-AuNPs were $97.8 \mu \mathrm{m}, 98.0 \mu \mathrm{m}, 108.9 \mu \mathrm{m}, 166.5 \mu \mathrm{m}$, and $155.9 \mu \mathrm{m}$, respectively. There was a significant difference in MSCs while being cultured in the PEG-HA-AuNP and PEG-AuNP groups, when compared to the control group $(p<0.01)$ (Figure 5D). It can be further inferred that both MC3TC cells and MSCs can promote cell attachment when cultured in nanomaterials. Moreover, this was also observed when cells cultured in either the PEG-HA-AuNP or PEG-AuNP group showed that the actin fiber elongation on MSCs was significantly higher than that on MC3T3 cells. The cell lengths of MC3T3 cells in the control (glass), PEG, PEG-HA, PEG-HA-AuNP, and PEG-AuNP groups were $107.8 \mu \mathrm{m}, 130.7 \mu \mathrm{m}, 129.3 \mu \mathrm{m}, 182.6 \mu \mathrm{m}$, and $244.3 \mu \mathrm{m}$, respectively, after $8 \mathrm{~h}$ of incubation. Additionally, in MSCs, after $8 \mathrm{~h}$ of incubation, the cell lengths in the control, PEG, PEG-HA, PEG-HA-AuNP, and PEG-AuNP groups were $72.5 \mu \mathrm{m}, 101.2 \mu \mathrm{m}$, $109.1 \mu \mathrm{m}, 129.6 \mu \mathrm{m}$, and $146.6 \mu \mathrm{m}$, respectively. As shown in these results, both the PEGHA-AuNP and PEG-AuNP groups significantly induced cell cytoskeleton change due to their production of more lamellipodia and filopodia, which can prominently induce cell actin fibers while exhibiting better extension properties. This, in turn, promotes a better adhesion and migration effect. Indeed, it was observed that the actin fiber extension of MC3T3 cells and MSCs while cultured on PEG-HA-AuNPs and PEG-AuNPs also had a similar extension tendency after $8 \mathrm{~h}$ of incubation when compared with the other groups (Figure S2A,B). It can be further inferred that both MC3TC cells and MSCs can promote cell attachment when cultured in nanomaterials. Moreover, this was also observed when cells cultured in either the PEG-HA-AuNP or PEG-AuNP group revealed that the actin fiber elongation on MSCs was significantly higher than that on MC3T3 cells (Figure S2C,D).

When an inflammatory response occurs, monocytes rapidly accumulate and differentiate into macrophages $(\sim 5 \mu \mathrm{m})$ within approximately $96 \mathrm{~h}(\sim 40$ to $45 \mu \mathrm{m})$ to trigger the immune response. The monocyte-to-macrophage transformation ratio on different materials after $96 \mathrm{~h}$ of incubation is presented in Figure 6A. The PEG-HA-AuNP and PEHAuNP groups exhibited a lower monocyte activation effect and lower conversion yield compared to the control group (TCPS), followed by the PEG and PEG-HA groups. Thus, it was demonstrated that PEG-HA-AuNPs and PEG-AuNPs can promote anti-inflammatory abilities, as well as offering better biocompatibility towards avoiding any foreign body reaction. Indeed, the CD68 (an indicator of macrophage markers) fluorescence intensity on PEG-HA-AuNPs ( 0.4-fold) and PEG-AuNPs ( 0.33-fold) showed a lower expression than that of the PEG ( 0.51-fold), PEG-HA ( 0.51-fold), and control (TCPS) groups $(p<0.05)$. The semi-quantified data were calculated using Image J software (Figure $6 \mathrm{C}$ ). The degree of platelet activation was observed through SEM analysis. As shown in Figure 6B, the number of adhered platelets was fewer, and they mostly represented a round morphology (non-activated form), while cell cultures in the PEG-HA-AuNP and PEG-AuNP groups did not. In contrast, regarding platelet cultures in the PEG, PEG-HA, and control groups, the adhered platelets increased and were subsequently exhibited as flattened (active form). The average degree of platelet adhesion ability in the PEG-HA-AuNP ( 0.28-fold) and PEGAuNP ( 0.19-fold) groups was compared to the PEG ( 0.99-fold), PEG-HA ( 0.54-fold), and control groups (glass) $(p<0.01)$ (Figure $6 \mathrm{D})$. Furthermore, the quantitative data also show that both PEG-HA-AuNPs and PEG-AuNPs can effectively suppress the number of monocytes and macrophages while also reducing the ratio of mononuclear cell conversion into macrophages (Figure S3A,C). 

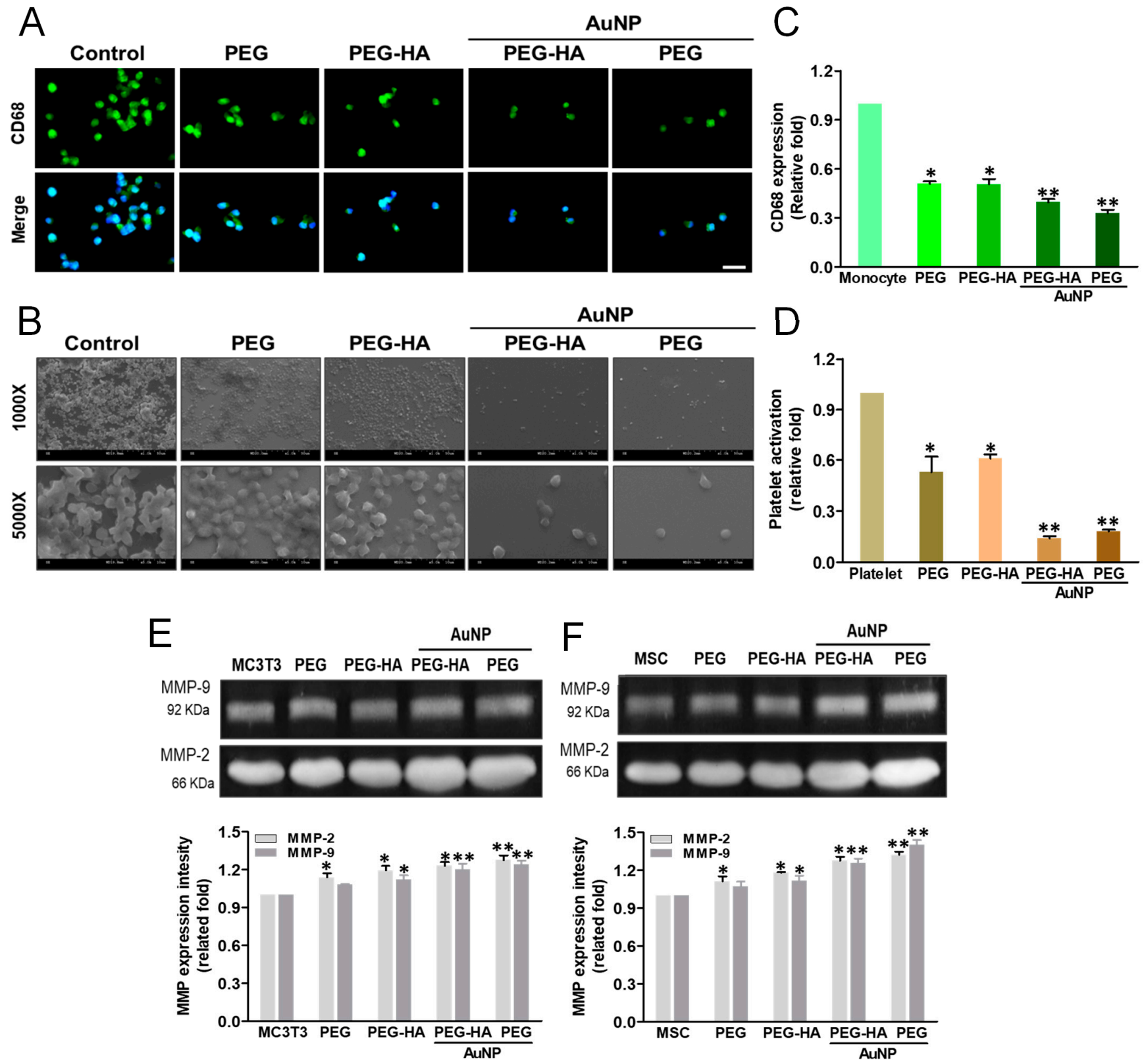

Figure 6. Biocompatibility assay. (A) The expression of CD68 for macrophages on different materials at $96 \mathrm{~h}$. Cells were immunostained by the primary anti-CD68 antibody and conjugated with the FITC-immunoglobulin secondary antibody (green color fluorescence). Cell nuclei were stained by DAPI (blue color fluorescence). Scale bar $=20 \mu \mathrm{m}$. (B) SEM images showing the adhesion and activation of human blood platelets on different materials. (C) CD68 expression was quantified based on fluorescence intensity. ${ }^{*} p<0.05$ : smaller than control (TCPS). (D) Quantification of the degree of platelet activation score. Data are the mean $\pm \mathrm{SD}(n=3) .{ }^{*} p<0.05 ;{ }^{* *} p<0.01$ : smaller than control (TCPS). The MMP-2/9 enzymatic activity of (E) MC3T3 cells and (F) MSCs was increased in the PEG-HA-AuNP and PEG-AuNP groups. Semi-quantitative measurement of MC3T3 cells and MSCs shows the expression level of MMP-2/9 protein for cells on different materials after $48 \mathrm{~h}$ of incubation. Semi-quantitative data in the graph represent the optical density (OD) of gelatinolytic bands. Data are the mean $\pm \mathrm{SD}(n=3){ }^{*} p<0.05 ;{ }^{* *} p<0.01$ : greater than control (TCPS).

The real-time images depicting cell migration were processed at both 24 and $48 \mathrm{~h}$ and observed under microscopy after Calcein AM staining. It was observed that with regard to the cell migration distance for MC3T3 cells (Figure S4A) and MSCs (Figure S4B) cultured on different materials, the PEG-HA-AuNP and PEG-AuNP groups were prominently higher than the PEG-HA, PEG, and control groups (TCPS) after 24 and $48 \mathrm{~h}$ of incubation. A similar result for MSCs while being cultured on different materials was also observed. 
The PEG-HA-AuNP and PEG-AuNP groups were prominently higher than the PEG-HA, PEG, and control groups (TCPS). Indeed, the quantification of the migration distance on MSCs cultured on the PEG-HA-AuNP $(21 \mu \mathrm{m})$ and PEG-AuNP $(23 \mu \mathrm{m})$ groups after $24 \mathrm{~h}$ and $48 \mathrm{~h}$ of incubation was prominently higher than that of MC3T3 cells cultured on the PEG-HA-AuNP $(13 \mu \mathrm{m})$ and PEG-AuNP $(15 \mu \mathrm{m})$ groups $(p<0.01)$ (Figure $\mathrm{S} 4 \mathrm{C})$. Indeed, the migration distance of MSCs cultured on the PEG-HA-AuNP and PEG-AuNP groups was $28 \mu \mathrm{m}$ and $32 \mu \mathrm{m}$, respectively, after $48 \mathrm{~h}$ of incubation; the migration distance of MC3T3 cells cultured on the PEG-HA-AuNP and PEG-AuNP groups was $31 \mu \mathrm{m}$ and $32 \mu \mathrm{m}$, respectively $(p<0.01)$ (Figure S4D). Based on these findings, it can be suggested that PEG-HA-AuNPs and PEG-AuNPs displayed a more prominent migration ability when compared to that of other materials. In particular, there was a significantly higher migration effect during the initial $24 \mathrm{~h}$ for MSCs. To explore whether MMP activity was induced by PEG-HA-AuNPs, both MC3T3 cells and MSCs were cultured on different materials. In the case of MC3T3 cells, the MMP-2 expression for PEG-HA-AuNPs and PEG-AuNPs was $\sim 1.2$-fold and $\sim 1.24$-fold, respectively, followed by PEG and PEG-HA, at $\sim 1.08$-fold and $~ 1.12$-fold $(p<0.01)$, respectively. Additionally, the MMP-9 expression on PEG-HAAuNPs and PEG-AuNPs was $~ 1.23$-fold and $~ 1.28$-fold, respectively, followed by PEG and PEG-HA, at $\sim 1.14$-fold and $~ 1.19$-fold $(p<0.01)$, respectively, after $48 \mathrm{~h}$ of incubation on MC3T3 cells (Figure 6E). Indeed, the MMP-2 expression of MSCs on PEG-HA-AuNPs and PEG-AuNPs was $~ 1.26$-fold and $~ 1.4$-fold, respectively, followed by PEG and PEG-HA, at $\sim 1.07$-fold and $\sim 1.12$-fold $(p<0.01)$, respectively. Moreover, the MMP-9 expression on PEG-HA-AuNPs and PEG-AuNPs was $\sim 1.28$-fold and $~ 1.32$-fold, respectively, followed by PEG and PEG-HA, at $~ 1.11$-fold and $\sim 1.18$-fold, respectively, after $48 \mathrm{~h}$ of incubation on MSCs $(p<0.01)$ (Figure 6F). These findings indicate that PEG-HA-AuNPs and PEG-AuNPs may lead to higher MMP expression levels in MSCs than those in MC3T3 cells.

We found that osteogenesis induction of MSCs occurred after 7, 14 and 21 days of incubation on different materials. The semi-quantification data show that the PEG-HAAuNP and PEG-AuNP groups were observed with slight mineral deposits as compared with the PEG, PEG-HA, and control groups (TCPS) on day 7. In addition, the mineral deposits increased in the PEG-HA-AuNP ( 1.77-fold) and PEG-AuNP ( 1.61-fold) groups when compared to the PEG ( 1.00-fold), PEG-HA ( 1.32-fold), and control groups (TCPS) over a longer period of time on day $21(p<0.01)$ (Figure $7 \mathrm{~A}, \mathrm{~B})$. Based on this finding, it can be suggested that PEG-HA-AuNPs and PEG-AuNPs may have the potential to enhance the differentiation capacity of MSCs into bone tissue. We also conducted real-time PCR analysis to investigate the Runx-2 (an osteogenic marker) gene expression level induced by different materials. In MSCs cultured on PEG-HA-AuNPs ( 3.46-fold) and PEG-AuNPs ( 3.58-fold), expression levels were significantly induced when compared with those for PEG ( 2.05-fold), PEG-HA ( 2.15-fold), and control group (TCPS) $(p<0.01)$ after 21 days of incubation (Figure 7C). The pattern of Runx-2 mRNA expression was similar to that taken from the observation of the ARS staining assay. In particular, PEG-HA-AuNPs and PEG-AuNPs had the potential to promote MSCs to differentiate into bone tissue. Osteocalcin (OCN), osteopontin (OPN), and alkaline phosphatase (ALP) were utilized as the osteoblastic differentiation markers. In our study, after induction of osteoblastic differentiation over 7, 14 and 21 days, real-time-PCR was performed for cells on different materials. PEG-HA-AuNPs showed the greatest upregulation of ALP at 2.26-fold, followed by OCN at 1.68-fold and OPN at 1.66-fold (Figure 7C). These results reveal that osteogenic marker genes in MSCs were upregulated by PEG-HA-AuNPs. 

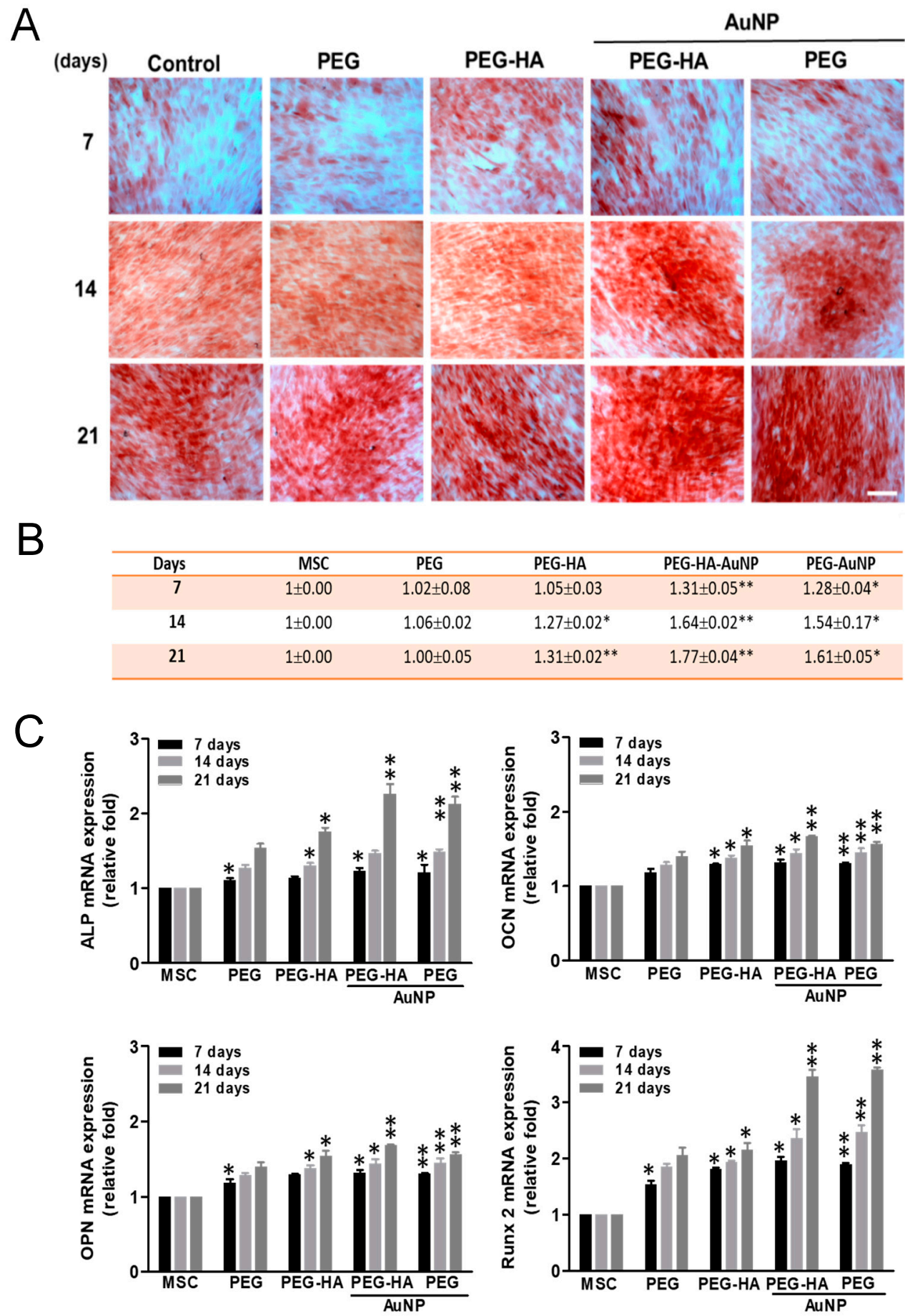

Figure 7. Osteogenic differentiation. (A) Osteogenic differentiation was confirmed by ARS staining of MSCs on different materials after 7, 14 and 21 days of incubation. Scale bar $=20 \mu \mathrm{m}$. (B) Semiquantification of osteoblastic differentiation by ARS staining. All images represent the mean \pm SD of three independent experiments. Data are the mean $\pm \operatorname{SD}(n=3) .{ }^{*} p<0.05 ;{ }^{* *} p<0.01$ : greater than control (TCPS). (C) Real-time PCR analysis of mRNA expression level for Runx-2, ALP, OCN, and OPN in MSCs after being cultured with different materials. GAPDH was used as an internal control. The results are represented as the ratio of Runx-2/GAPDH signals for each condition, normalized to control. Data are the mean $\pm \mathrm{SD}(n=3)$. ${ }^{*} p<0.05 ;{ }^{* *} p<0.01$ : greater than control (TCPS).

Bone vasculature plays an important role in bone development, remodeling, and homeostasis. Angiogenesis has been induced by the endothelial nitric oxide synthase gene through vascular endothelial growth factor expression in a rat ischemia model. Overall, 
intramuscular injection of the eNOS plasmid induced therapeutic angiogenesis in a rat ischemic hindlimb model, offering a potential therapy for peripheral arterial disease. The stimulation of angiogenesis caused by NO may be due to the upregulation of local VEGF expression [63]. We analyzed the VEGF expression of MSCs through an ELISA assay. After MSC growth on different materials over $48 \mathrm{~h}$ of incubation, VEGF expression was significantly induced by both PEG-HA-AuNPs ( 1.37-fold) and PEG-AuNPs ( 1.32-fold), as compared to the control group $(p<0.01)$ (Figure $8 \mathrm{~A})$. These results demonstrate that the nanocomposites may promote eNOS production and VEGF expression as inferred by the endothelialization capacity for bone tissue regeneration. We also investigated the macrophage-mediated cytokine expression levels on different materials. When RAW 264.7 cells were cultured on PEG-HA-AuNPs and PEG-AuNPs, the concentrations of IL-1 $\beta$, IL-6, and TNF- $\alpha$ decreased over varying times of incubation. However, in the groups of PEG-HA-AuNPs and PEG-AuNPs, the levels of the anti-inflammatory cytokine IL-10 significantly increased compared to those in the other groups (Figure 8B). The schematic diagram shows that PEG-HA-AuNPs with MSCs induced better angiogenic and osteogenic differentiation. After combining with PEG-HA-AuNPs, the expression of CD 86 was decreased. In contrast, CD 163 expression was increased. This result indicates that PEGHA-AuNPs may inhibit the inflammatory response. Moreover, PEG-HA-AuNPs effectively promoted endothelialization, leading to a higher expression of CD 31. They also induced the expression of the Runx-2 gene, which enhanced the differentiation of MC3TC cells into osteocytes. The former was linked to angiogenesis, while the latter was related to osteogenesis. The above evidence supports the theory that PEG-HA-AuNPs could become an outstanding biomaterial for bone tissue regeneration (Figure $8 \mathrm{C}$ ).

The foreign body reaction from the different materials was determined through subcutaneous implantation over the course of one month to assess both the biocompatibility and inflammatory response. The characteristics of the fibrous capsule on both PEG-HAAuNPs ( 0.47-fold) and PEG-AuNPs ( 0.47-fold) exhibited less thickness, followed by PEG ( 0.81-fold) and PEG-HA ( 0.91-fold), when compared to the control group $(p<0.01)$ (Figure 9A,D). Moreover, in order to further confirm the endothelialization ability of MSCs on PEG-HA-AuNPs in vivo, materials were subjected to subcutaneous implantation for one month. IHC staining showed that CD31-positive cells abundantly occurred with PEGHA-AuNPs ( 2.09-fold) and PEG-AuNPs ( 1.69-fold), followed by PEG ( 1.31-fold) and PEG-HA ( 0.99-fold), when compared to the control group $(p<0.01)$. It can be suggested that PEG-HA-AuNPs displayed a superior endothelialization capacity when compared to the other test groups (Figure 9B,E). Furthermore, in order to confirm the foreign body reaction caused by different materials, we further stained the infiltration of CD45 (a marker of leukocytes) in order to assess the foreign body reaction to the materials after one month of subcutaneous implantation into rats. Indeed, the CD45 fluorescence intensity on PEGHA-AuNPs ( 0.32-fold) and PEG-AuNPs ( 0.29-fold) showed a lower expression than that on the PEG ( 0.8-fold), PEG-HA ( 1.12-fold), and control (TCPS) groups $(p<0.01)$. The semi-quantified data were calculated using Image J software (Figure $9 \mathrm{C}, \mathrm{F}$ ). This result suggests that these materials may be safe for biomaterial application purposes. 
A
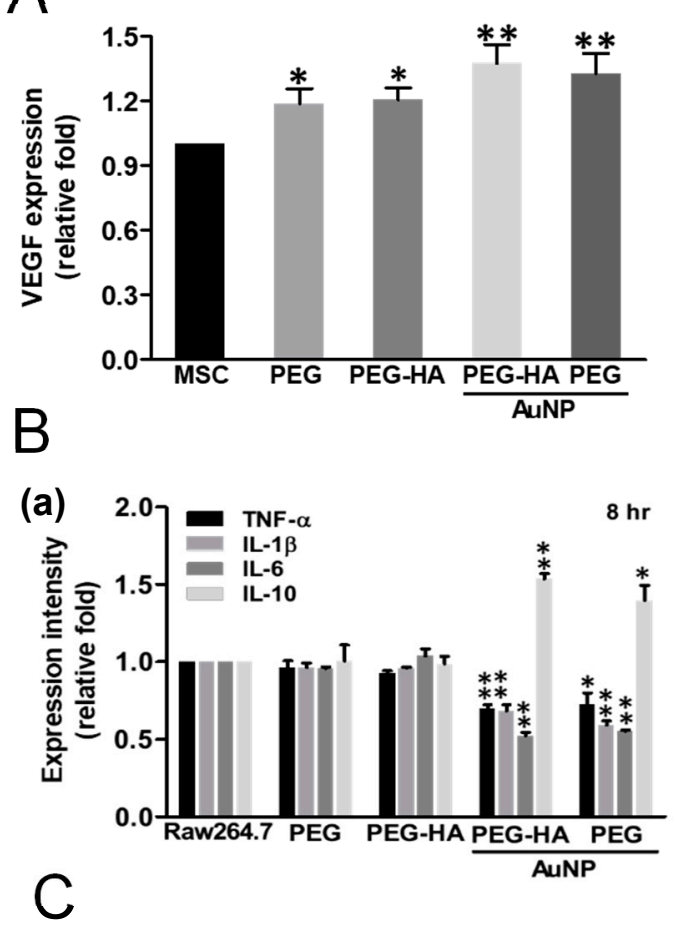

(b)

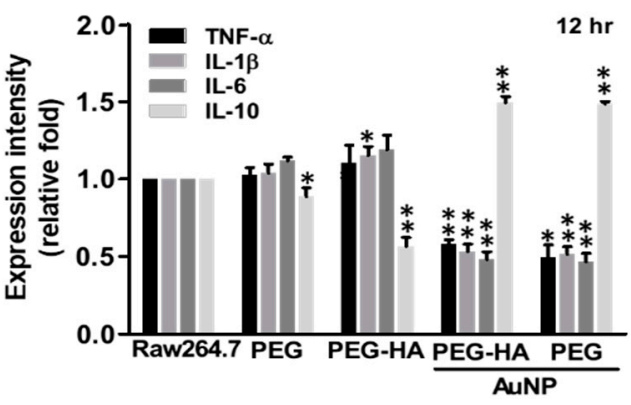

(c)

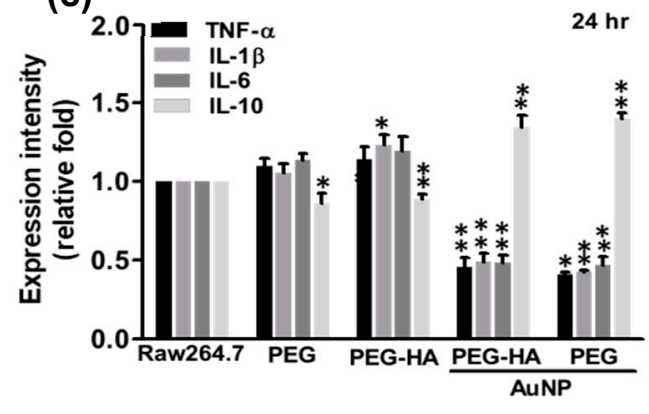

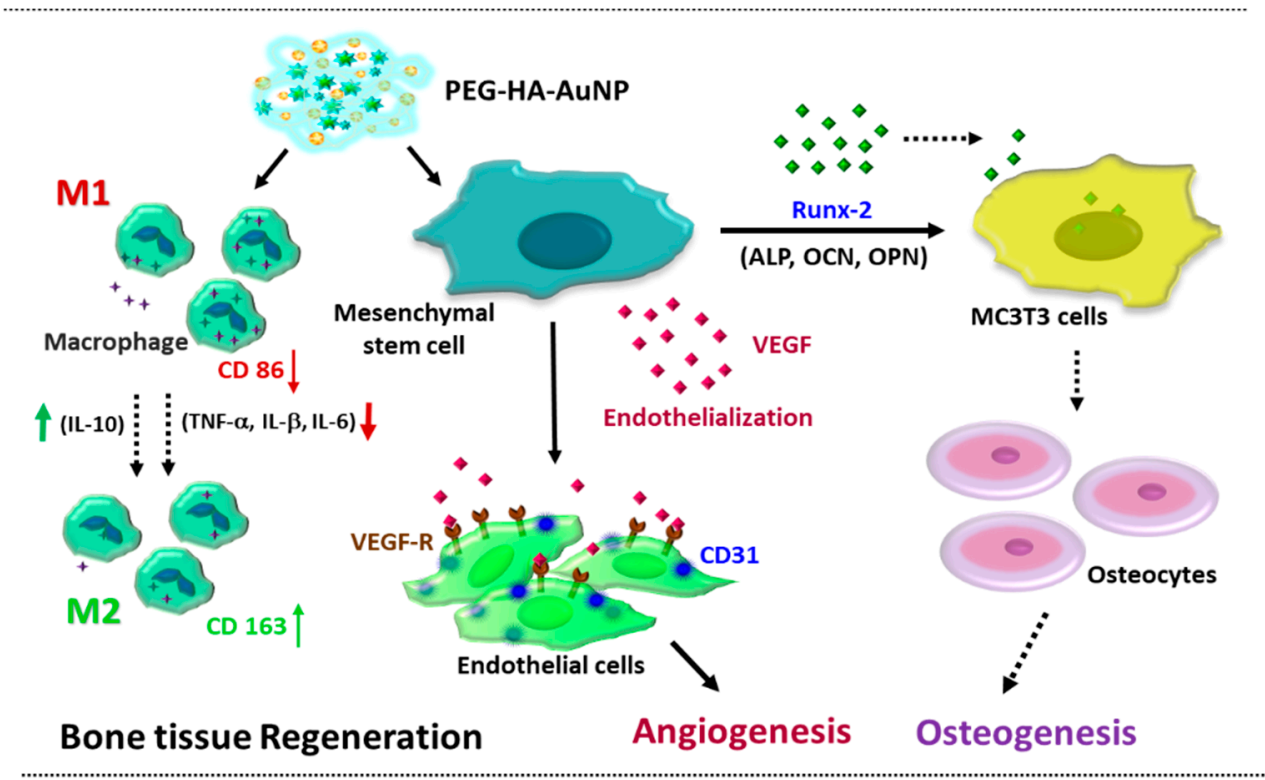

Figure 8. Assessment of the immune response and angiogenesis of MSCs on different materials. (A) Measurement by ELISA assay shows significantly increased VEGF protein expressions for MSCs after culture on different materials after $48 \mathrm{~h}$ of incubation. Data are mean \pm SD. ${ }^{*} p<0.05:{ }^{* *} p<0.01$ : greater than TCPS. (B) ELISA results for inflammatory cytokines: (a) $8 \mathrm{~h},(\mathbf{b}) 12 \mathrm{~h}$, and (c) $24 \mathrm{~h}$ of production by RAW264.7 cells. ${ }^{*} p<0.05$; ${ }^{* *} p<0.01$. (C) Scheme illustrates PEG-HA-AuNPs' prominent superior biological and biocompatibility performance, which may account for the induction of the better differentiation ability into bone tissue of MSCs for this substrate. Schematic diagram shows that PEG-HA-AuNPs with MSCs induced better angiogenic and osteogenic differentiation. After combining with PEG-HA-AuNPs, the expression of CD 86 was decreased. In contrast with CD 86, CD 163 expression was increased. This result indicates that PEG-HA-AuNPs could inhibit the inflammation response. Moreover, PEG-HA-AuNPs effectively promoted endothelialization, leading to a higher expression of CD 31; they also induced the expression of the Runx-2 gene, which caused MC3T3 cells to differentiate into osteocytes. The former was linked to angiogenesis, and the latter was related to osteogenesis. The above evidence shows that PEG-HA-AuNPs could become an outstanding biomaterial for bone tissue regeneration. 
A
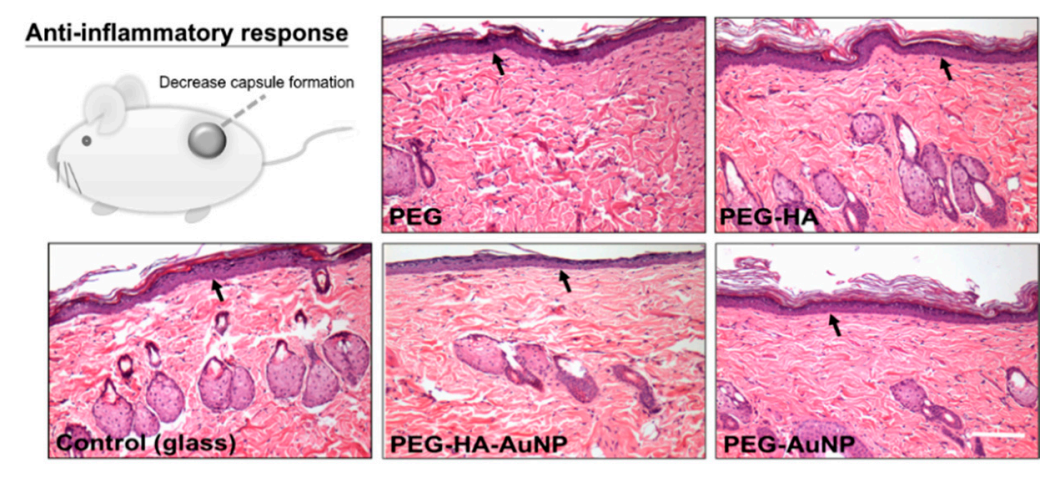

B
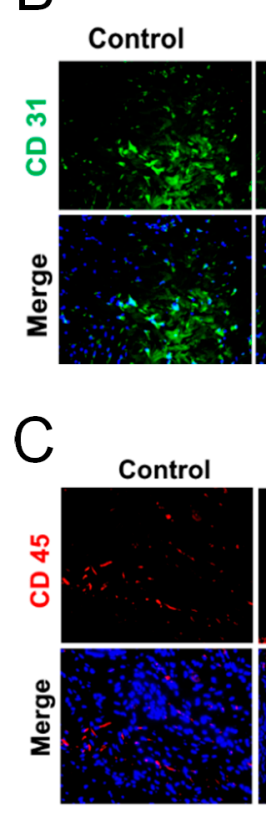
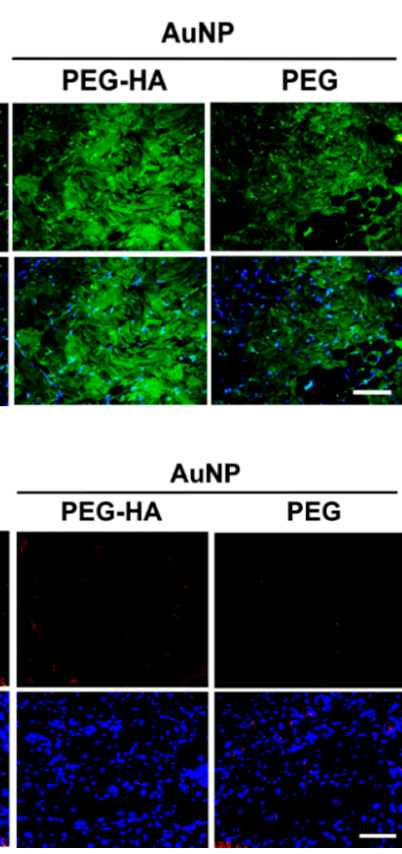

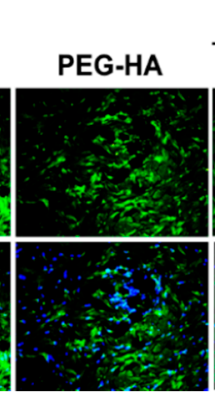

D
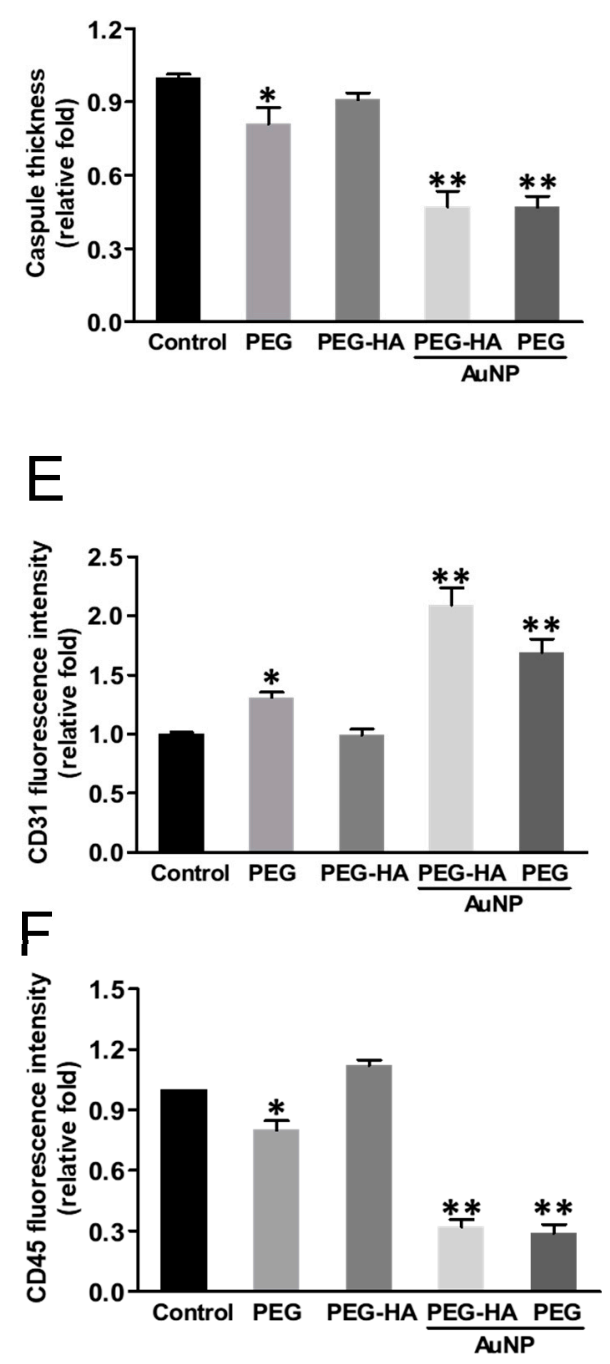

Figure 9. Evaluation of foreign body response of different substrates after subcutaneous implantation. Histology of H\&E-stained sections and IHC staining after implantation of material for 4 weeks. (A) FBR is exhibited by the capsule thickness (arrows) based on the histology examination. The scale bar is $100 \mu \mathrm{m}$. (B) Immunofluorescence staining of CD31 (marker of endothelialization), and (C) CD45 (marker of immunoflammation) in response to the implant materials. The scale bar is $100 \mu \mathrm{m}$. Quantification of (D) capsule thickness, and (E) CD31 and (F) CD45 fluorescence intensities. Data are mean \pm SD. ${ }^{*} p<0.05,{ }^{* *} p<0.01$ : greater than control (glass). The number of rats was $5(n=5)$.

Tissue samples were analyzed for collagen deposition and collagen concentrations as a measure of tissue fibrosis. Masson's trichrome staining revealed significant collagen deposition in response to the control-treated group (glass). The intensity of collagen deposition in the PEG-HA-AuNP ( 0.72-fold) and PEG-AuNP ( 0.70-fold) groups was prominently lower than that in the PEG group $(\sim 0.92$-fold $)$, followed by the PEG-HA group ( 1.45-fold), when compared to the control group $(p<0.01)(p<0.01)$ (Figure 10A,D). Regarding this finding, the subcutaneous implantation of PEG-HA-AuNPs and PEGAuNPs into rats reduced the amount of collagen deposition as well as attenuating the tissue fibrosis effect, particularly in the PEG-AuNP and PEG-AuNP-HA test groups. CD45 plays complex roles in both $\mathrm{T}$ cell and $\mathrm{B}$ cell antigen receptor signal transduction [64]. Subsequently, we further assessed the macrophage maker expression (M1: CD86, M2: CD163) in order to evaluate the inflammatory index of materials subcutaneously implanted for a period of one month. Indeed, CD86 expression was lower for PEG-HA-AuNPs 
( 0.37-fold) and PEG-AuNPs ( 0.45-fold), followed by PEG-HA ( 0.82-fold) and PEG $(\sim 0.69$-fold), when compared to the control group $(p<0.01)$ (Figure 10B,E). Indeed, CD163 showed a more prominent expression on PEG-HA-AuNPs ( 1.93 -fold) and PEG-AuNPs ( 1.81-fold), followed by PEG-HA ( 1.15-fold) and PEG ( 1.47-fold), when compared to the control group $(p<0.01)$. Based on this finding, it can be suggested that both PEGHA-AuNPs and PEG-AuNPs may significantly promote the anti-inflammatory response in vivo (Figure 10C,F). The TUNEL test is an appropriate test for the biosafety concern surrounding these materials. The number of apoptotic cells was not obviously observed in all groups. There was not a significant difference in all test groups (Figure S5).

A

Anti-fibrosis ability
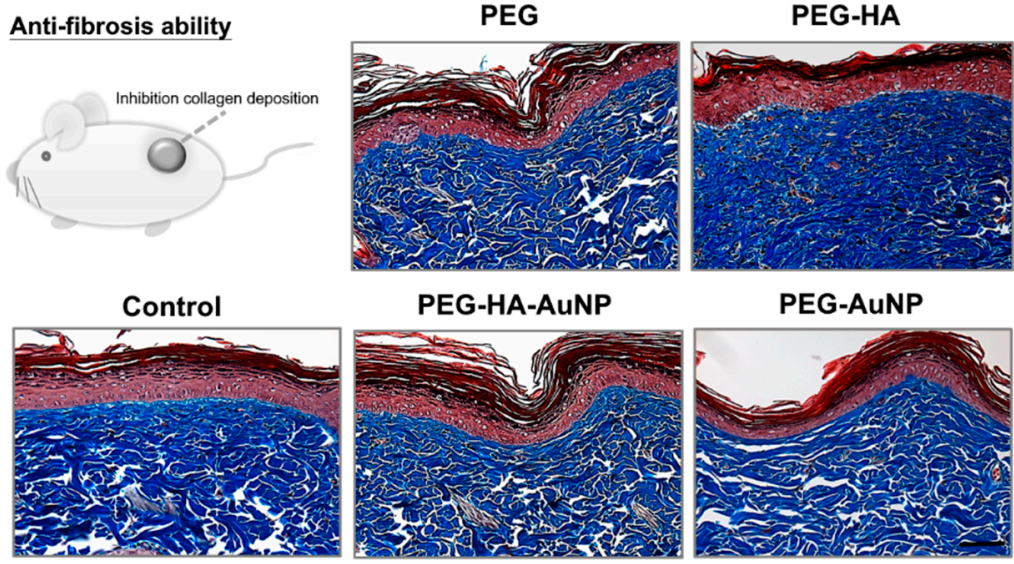

PEG-HA-AuNP

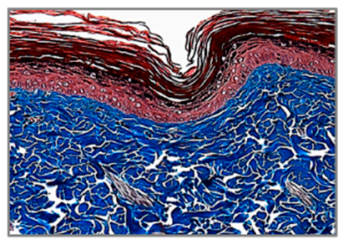

PEG-AuNP

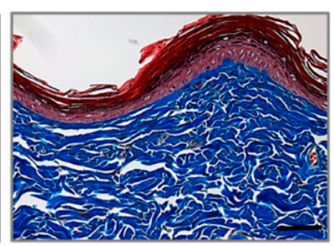

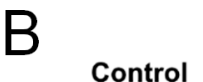
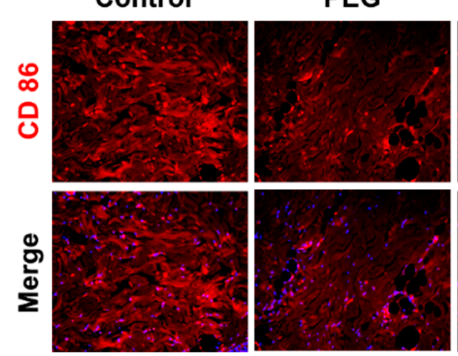

C
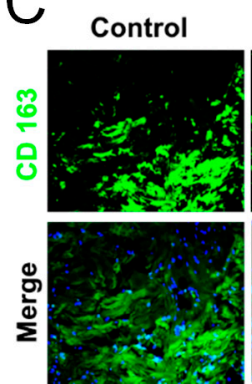

PEG
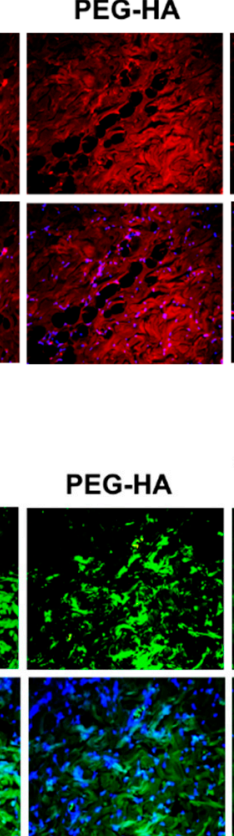

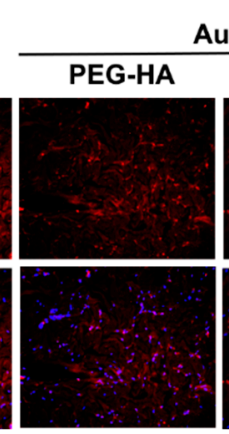

AuNP

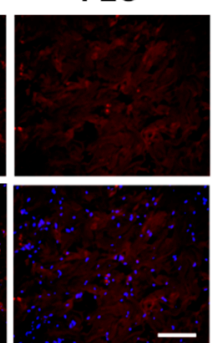

AuNP
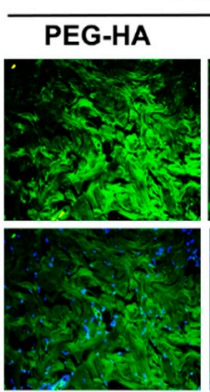

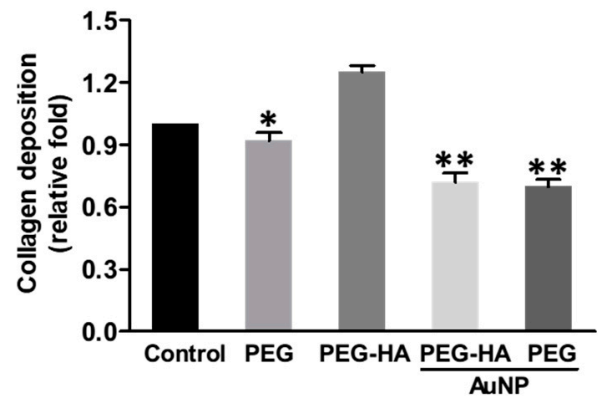

$\mathrm{D}$
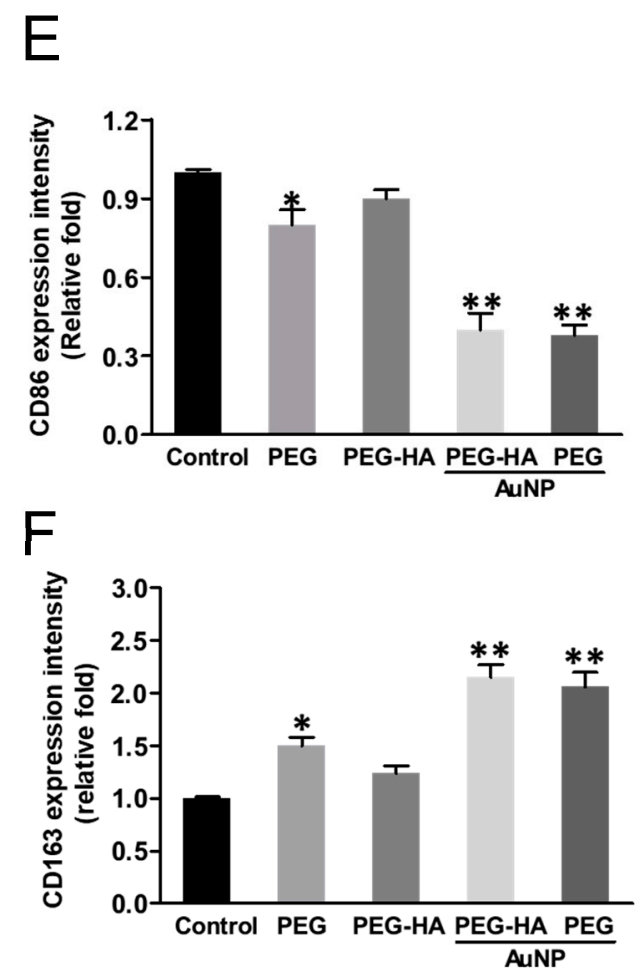

Figure 10. Masson's trichrome staining of (A) collagen deposition (blue color) of rat femoral artery subcutaneously implanted in an SD rat assessed the immune response of different materials at 4 weeks. Immunofluorescence staining images (marker of macrophages) in response to the implant materials. (B) CD86 (M1) = red color, $($ C) CD163 (M2) = green color. The scale bar represents $100 \mu \mathrm{m}$. Quantification of (D) collagen deposition, and (E) CD86 and (F) CD163 fluorescence intensities. Data are mean \pm SD. ${ }^{*} p<0.05,{ }^{* *} p<0.01$ : smaller or greater than control group (glass). The number of rats was $5(n=5)$. 


\section{Discussion}

Gold nanoparticles (AuNPs) have inert properties due to their excellent performance. As discussed in our previously published papers, when there is incorporation of an appropriate concentration of AuNPs into different types of polymers, leading to the formation of nanocomposites, it can cause superior biocompatibility and biological performance by nanotopography cues, as well as producing a biomimic interface, subsequently changing the cell behavior. The impressive capacity of nanocomposites may be attributed to their superior biocompatibility, which is not only due to their lower monocyte counts and platelet activation but also their higher cell growth, migration, and differentiation capabilities $[59,65,66]$.

Hydrogels as stem cell scaffolds are a promising method for conquering initial cell loss and manipulating cell function post-transplantation. Scaffold degradation is required for downstream cell differentiation and functional tissue integration for determining results of therapies [67]. Additionally, electrospinning supplies various techniques for preparing micro- or nanoscopic fibers with matrices. Polymer materials which were produced through electrospinning had a high surface-to-volume ratio and pore interconnectivity [68]. The above research can be considered when seeking to provide a more effective and practical treatment and solution to bone tissue engineering. Polyethylene glycol (PEG) is modified into a variety of biomaterials in order to improve biocompatibility due to its superior safety and efficacy abilities [69]. MSCs are very useful in regenerative medicine due to their potential for the regeneration of different types of damaged tissues, particularly bone tissue $[19,70]$. Another study in the available literature illustrated that the differentiation of MC3T3-E1 pre-osteoblasts promotes growth in hydrogels through hydroxyapatite nanoparticles [71]. Higher adherence efficiency, as well as the induction of higher ALP activity, in human osteoblast-like MG-63 cells subsequently occurred. An HA-PEG composite involved chains of disuccinimidyl tartrate in order to obtain a PEG hydrogel. Therefore, HA-PEG composites offer potential as a bone substitute application [72].

Based on these findings, the use of PEG-HA-AuNP nanocomposites provides a microenvironment which mimics the microenvironment for differentiation into the bone tissue of MSCs. The elastic properties of the matrix correlate with stem cell differentiation (Figure 2), where they can guide different cell behavior changes via mechanical attraction cues. Moreover, the surface roughness of the original PEG and PEG-HA was approximately $\sim 0.31 \mathrm{~nm}$ and $\sim 5.4 \mathrm{~nm}$, respectively, with the roughness of PEG-HA-AuNPs and PEGAuNPs significantly reduced to $\sim 0.5$ and $\sim 0.97 \mathrm{~nm}$, respectively, after the addition of an optimal concentration of AuNPs (Figure 2). Therefore, it can be inferred that an appropriate concentration of AuNPs can significantly improve the roughness of the PEG-HA material surface to become smoother and more uniform. After performing calculations, the Young modulus values for PEG-HA-AuNPs and PEG-AuNPs were lower than those for PEG and PEG-HA (Figure 2), demonstrating that the properties of PEG-HA-AuNPs and PEG-AuNPs were softer and had decreased through the incorporation of an optimal concentration of AuNPs, as seen in the AFM analysis. Based on the above results, it can be suggested that the specific nanotopography features of both PEG-HA-AuNPs and PEG-AuNPs can be effective in guiding cells towards producing better attachment, growth, migration, and differentiation. Meanwhile, much published literature has proved that most cells prefer to adhere to the hydrophilic surface of biomaterials [18]. Our results found that when there was cell growth on PEG-HA-AuNPs and PEG-AuNPs, the hydrophilicity properties significantly increased when compared to both the PEG-HA and control (TCPS) groups (Figure 5). Until now, much research has indicated that when stem cells are in the differentiation stage, the activation of MMP2/9 will be triggered and therefore effectively promote stem cells towards migration and differentiation events through SDF- $1 \alpha /$ CXCR4 signaling pathways [53]. Interestingly, we also observed the migration rate of MSCs and saw better migration efficiency when compared to MC3T3 cells after $24 \mathrm{~h}$ of incubation, particularly for the PEG-HA-AuNP and PEG-AuNP groups. Therefore, we deduced that 
nanocomposites can stimulate MMP expression, which, in turn, may promote superior differentiation for the osteogenesis of MSCs.

Gold-hydroxyapatite nanostructures potentiate the osteogenic differentiation of MSCs, as evidenced by a prominent over-expression of Runt-related transcription factor 2 (Runx-2) and Bone Morphogenetic Protein 2 (BMP-2) genes after 7 days of incubation [73]. As evidenced from this result, PEG-HA-AuNPs and PEG-AuNPs can significantly induce Runx-2 gene expression (Figure 7), as well as producing the osteogenesis of MSCs after being cultured on materials for 7, 14 and 21 days of incubation (Figure 7). Moreover, the incorporation of AuNPs, which seemed to stimulate the osteoinductive capability of HA-AuNP nanomaterials, may modulate the Wnt/ $\beta$-catenin signaling pathway [74]. Therefore, elucidating the molecular mechanism of PEG-HA-AuNPs and PEG-AuNPs has the potential to promote superior biocompatibility, biological capability, and differentiation capacity, although this still requires further exploration in the future.

It is well known that angiogenesis plays an important physiological function in the process of bone tissue regeneration [71]. New blood vessel formation is essential during both primary bone development and fracture repair in adults. Both bone repair and remodeling involve the activation and complex interaction between angiogenic and osteogenic pathways. Interestingly, studies have demonstrated that angiogenesis precedes the onset of osteogenesis [75]. We have previously demonstrated that the addition of gold nanoparticles in polyurethane can induce changes in nanomorphology and effectively activate the expression of eNOS and VEGF, thereby promoting vascular endothelialization, angiogenesis, and endothelial tissue repair [65]. Bone vasculature plays a vital role in bone development, remodeling, and homeostasis. New blood vessel formation is crucial during both primary bone development and fracture repair in adults. Both bone repair and bone remodeling involve the activation and complex interaction between the angiogenic and osteogenic pathways. Angiogenesis precedes the onset of osteogenesis [75]. The stimulation of angiogenesis by NO may be due to the upregulation of local VEGF [61]. In order to better understand whether the materials had a promoting effect on the production of eNOS by endothelial cells, we plated endothelial cells on the materials for $48 \mathrm{~h}$ and found that PEG-HA-AuNPs and PEG-AuNPs significantly increased eNOS production and promoted endothelialization (Figure 8). From in vivo animal experiments, we discovered that after materials were subcutaneously implanted into rats for a period of one month, the PEG-HA-AuNP and PEG-AuNP groups effectively promoted a higher CD31 expression level (as an endothelial marker) than that in the PEG and PEG-HA groups (Figure 9). Additionally, successfully inhibited lipopolysaccharide (LPS)-induced interleukin-6 (IL6) and inducible nitric oxide synthase (iNOS) secretion by M1 macrophages facilitates osteogenesis, which was investigated on a rat calvarial defect model [76]. Therefore, in order to prove whether PEG-HA-AuNP nanocomposites can be safely used in the human body, we further detected the foreign body reaction (FBR) through HE staining. As shown in this study, both the PEG-HA-AuNP and PEG-AuNP groups were able to effectively reduce the production of capsules after subcutaneously implanting materials for a period of one month (Figure 9).

Several studies have shown that pro-inflammatory cytokines are accompanied by the sensitization of cells such as neutrophils, macrophages, and endothelial cells. RAW 264.7 cells are commonly used as macrophages to show phenotype alterations in response to environmental changes $[77,78]$. Thus, we selected RAW 264.7 cells in the present study to investigate inflammatory cytokine secretion in different materials (Figure 8). Additionally, we also doubly confirmed the anti-inflammatory response of nanocomposites through IHC staining. The activation of CD86 (as a marker of M1) was decreased, while the expression of CD163 (as a marker of M2) was increased in both the PEG-HA-AuNP and PEG-AuNP groups. Based on these findings, we suggest that PEG-HA-AuNPs and PEG-AuNPs potentially inhibited the inflammation process and anti-fibrosis ability in vivo (Figure 10).

One study has pointed out that converting collagen and HA into bone biomaterials can effectively promote bone tissue mineralization, as well as inducing the regeneration of bone 
when there is an addition of iron and manganese nanoparticles [79]. Similar to this finding, our research also demonstrates that the modification of HA with PEG and the incorporation of an optimal concentration of AuNPs not only effectively promote the potential of stem cells to differentiate into bone tissue but also display a strong biocompatibility ability and superior functionality. Indeed, another published report also demonstrated that the modification of Ti biomaterials with gold nanoparticles can effectively promote bone regeneration in a rabbit model [80]. Meanwhile, a novel triblock PEG-PCL-PEG copolymer composed of collagen and nano-hydroxyapatite (n-HA) (PECE/Collagen/n-HA) offered better biocompatibility as well as superior performance in osteogenesis, leading to a bone tissue healing process in New Zealand white rabbits after being implanted over 4-, 12-, and 20-week periods [35]. In relation to this finding, our research also found that incorporating AuNPs into PEG-HA can effectively promote MSCs to differentiate into bone tissue. Therefore, we will use this PEG-HA-AuNP nanocomposite as a model system to further confirm whether PEG-HA-AuNPs also provide better repair potential for bone tissue regeneration in vivo in our future work.

Regarding these findings, it can be suggested that the PEG-HA-AuNP and PEGAuNP groups were able to provide a suitable biocompatibility property for cell attachment, proliferation, and proper differentiation. The findings also prove that the addition of an optimal concentration of AuNPs onto the PEG-HA material surface significantly promoted the efficiency of osteogenic differentiation. Our results reveal that, due to their proper biocompatibility, as well as superior biological capability, PEG-HA-AuNPs and PEGAuNPs may be considered as ideal nano-biomaterials and further applied towards the repair of bone tissue in the future.

\section{Conclusions}

In this report, we outlined how a concise surface modification technique was applied to produce PEG-HA-AuNP nanocomposites. This approach successfully constructed a PEG-HA-AuNP model, in which eminent materials were optimized to achieve advanced biocompatibility and biological capability, as observed in the in vitro assay. This study also illustrated that PEG-HA-AuNPs can induce MSCs via MMP activation and subsequently trigger a migration effect, as well as guiding the osteogenic differentiation of MSCS on this material. Simultaneously, the PEG-HA-AuNP nanocomposites effectively improved the endothelialization capability, anti-inflammatory response, and anti-fibrosis ability after subcutaneous implantation of PEG-HA-AuNPs into a rat after 1 month, making them a potential candidate for surface modification of biomaterials in bone tissue regeneration.

Supplementary Materials: The following are available online at https:/ /www.mdpi.com/article/10 .3390/biomedicines9111632/s1, Figure S1. Characterization of MSCs using flow cytometry analysis. (A) Cells were harvested and incubated with the respective antibody conjugated with fluorescein isothiocyanate (FITC) and/or phycoerythrin (PE). The indicated markers: CD14-FITC, CD34-FITC, CD45-FITC, CD44-PE, CD73-PE, and CD90-PE (BD Pharmingen, USA). Filled area represents isotype controls. (B) The FACS results of negative markers: CD14, CD34, and CD45 expression. (C) The FACS data of positive markers: CD44, CD73, and CD90 expression. Figure S2. Cytoskeleton and cell morphology by rhodamine phalloidin staining of (A) MC3T3 cells and (B) MSCs for actin fiber extension on different materials after $8 \mathrm{~h}$ of incubation under fluorescence microscopy analysis. Scale bar $=50 \mu \mathrm{m}$. Arrows indicate filopodia (green color) and lamellipodia (red color). The actin fiber extension in length quantified by Image J software in (C) MC3T3 cells and (D) MSCs on different materials after $8 \mathrm{~h}$ is shown. Actin fiber length elongation was significantly observed in the PEG-HAAuNP and PEG-AuNP test groups compared with the other groups. Data are mean $\pm \operatorname{SD}(n=3)$. ${ }^{*} p<0.05 ;{ }^{* *} p<0.05$. Scale bar $=50 \mu \mathrm{m}$. Data are the mean $\pm \mathrm{SD}(n=3)$. Figure S3. Quantification on the activation degree of monocytes and platelets. Number of (A) monocytes and (B) macrophages, and $(\mathrm{C})$ conversion ratio of monocytes to macrophages. Data are the mean $\pm \operatorname{SD}(n=3) .{ }^{*} p<0.05$; ** $p<0.01$ : smaller than control (TCPS). Figure S4. Migration of (A) MC3T3 cells and (B) MSCs on different materials during a period of 24 to $48 \mathrm{~h}$. Cell migration into the gap zone area was monitored by fluorescence microscopy. Cells were stained by Calcein AM $(2 \mu \mathrm{M})$ prior to examination. Scale 
bar $=500 \mu \mathrm{m}$. Quantification of the fluorescence intensity of (C) MC3T3 cells and (D) MSCs by Image J software. Data are the mean $\pm \mathrm{SD}(n=3) .{ }^{*} p<0.05 ;{ }^{* *} p<0.01$ : greater than control (TCPS). Data were obtained from three different experiments. Figure S5. (A) Representative images of immunofluorescence staining for TUNEL-positive nuclei in different groups ( $20 \times$ magnification). (B) Quantification of TUNEL-positive cells shows a lower number in the treated group as compared to the control group. Scale bar $=100 \mu \mathrm{m}$. The number of rats was $5(n=5)$. Table S1. XPS analysis of PEG and PEG composites.

Author Contributions: Conceptualization, C.-C.S. and H.-S.H.; data curation, C.-C.S., C.-A.Y., H.-C.C., C.-M.T., Y.-C.Y. and H.-H.H.; formal analysis, S.-h.H., K.-B.C., C.-A.Y., H.-C.C., C.-M.T. and Y.-C.Y.; funding acquisition, H.-S.H.; investigation, S.-h.H. and H.-S.H.; methodology, C.-C.S. and H.-C.C.; resources, H.-H.H.; software, C.-A.Y.; supervision, H.-S.H.; validation, C.-C.S.; visualization, H.-S.H.; writing-original draft, C.-C.S. and K.-B.C.; writing—review and editing, S.-h.H. All authors have read and agreed to the published version of the manuscript.

Funding: This work was supported by grants from the China Medical University Hospital (DMR105-057) and the Ministry of Science and Technology (MOST 109-2314-B-075B-011-MY3).

Institutional Review Board Statement: Not applicable.

Informed Consent Statement: Not applicable.

Data Availability Statement: Data are contained within the article.

Acknowledgments: Murine macrophage RAW 264.7 cells were kindly provided by Professor Hui-Jen Chen (China Medical University, Taiwan).

Conflicts of Interest: The authors have declared that no competing interest exists.

\section{References}

1. Zhu, T.; Cui, Y.; Zhang, M.; Zhao, D.; Liu, G.; Ding, J. Engineered three-dimensional scaffolds for enhanced bone regeneration in osteonecrosis. Bioact. Mater. 2020, 5, 584-601. [CrossRef] [PubMed]

2. Zhao, D.; Zhu, T.; Li, J.; Cui, L.; Zhang, Z.; Zhuang, X.; Ding, J. Poly (lactic-co-glycolic acid)-based composite bone-substitute materials. Bioact. Mater. 2021, 6, 346-360. [CrossRef] [PubMed]

3. Qiu, H.; Guo, H.; Li, D.; Hou, Y.; Kuang, T.; Ding, J. Intravesical Hydrogels as Drug Reservoirs. Trends Biotechnol. 2020, 38, 579-583. [CrossRef] [PubMed]

4. Le, H.; Xu, W.; Zhuang, X.; Chang, F.; Wang, Y.; Ding, J. Mesenchymal stem cells for cartilage regeneration. J. Tissue Eng. 2020, 11, 2041731420943839. [CrossRef]

5. Cui, L.; Zhang, J.; Zou, J.; Yang, X.; Guo, H.; Tian, H.; Zhang, P.; Wang, Y.; Zhang, N.; Zhuang, X.; et al. Electroactive composite scaffold with locally expressed osteoinductive factor for synergistic bone repair upon electrical stimulation. Biomaterials 2020, 230, 119617. [CrossRef]

6. Kim, S.S.; Sun Park, M.; Jeon, O.; Yong Choi, C.; Kim, B.S. Poly(lactide-co-glycolide)/hydroxyapatite composite scaffolds for bone tissue engineering. Biomaterials 2006, 27, 1399-1409. [CrossRef] [PubMed]

7. Li, J.; Zheng, Q.; Guo, X.; Zou, Z.; Liu, Y.; Lan, S.; Chen, L.; Deng, Y. Bone induction by surface-double-modified true bone ceramics in vitro and in vivo. Biomed. Mater. 2013, 8, 035005. [CrossRef] [PubMed]

8. Garai, S.; Sinha, A. Biomimetic nanocomposites of carboxymethyl cellulose-hydroxyapatite: Novel three dimensional load bearing bone grafts. Colloids Surf. B Biointerfaces 2014, 115, 182-190. [CrossRef]

9. Moore, W.R.; Graves, S.E.; Bain, G.I. Synthetic bone graft substitutes. ANZ J. Surg. 2001, 71, 354-361. [CrossRef]

10. Wahl, D.A.; Czernuszka, J.T. Collagen-hydroxyapatite composites for hard tissue repair. Eur. Cell Mater. 2006, 11, 43-56. [CrossRef]

11. Di Toro, R.; Betti, V.; Spampinato, S. Biocompatibility and integrin-mediated adhesion of human osteoblasts to poly(DL-lactideco-glycolide) copolymers. Eur. J. Pharm. Sci. 2004, 21, 161-169. [CrossRef]

12. Wang, G.; Liu, S.J.; Ueng, S.W.; Chan, E.C. The release of cefazolin and gentamicin from biodegradable PLA/PGA beads. Int. J. Pharm. 2004, 273, 203-212. [CrossRef] [PubMed]

13. Clèries, L.; Fernández-Pradas, J.M.; Morenza, J.L. Behavior in simulated body fluid of calcium phosphate coatings obtained by laser ablation. Biomaterials 2000, 21, 1861-1865. [CrossRef]

14. Ramesh, N.; Moratti, S.C.; Dias, G.J. Hydroxyapatite-polymer biocomposites for bone regeneration: A review of current trends. J. Biomed. Mater. Res. B Appl. Biomater. 2018, 106, 2046-2057. [CrossRef]

15. Webster, T.J.; Ergun, C.; Doremus, R.H.; Siegel, R.W.; Bizios, R. Specific proteins mediate enhanced osteoblast adhesion on nanophase ceramics. J. Biomed. Mater. Res. 2000, 51, 475-483. [CrossRef]

16. Murugan, R.; Ramakrishna, S. Development of nanocomposites for bone grafting. Compos. Sci. Technol. 2005, 65, 2385-2406. [CrossRef] 
17. Costa-Rodrigues, J.; Carmo, S.; Perpétuo, I.P.; Monteiro, F.J; Fernandes, M.H. Osteoclastogenic differentiation of human precursor cells over micro- and nanostructured hydroxyapatite topography. Biochim. Biophys. Acta 2016, 1860, 825-835. [CrossRef]

18. Wang, P.; Yu, T.; Lv, Q.; Li, S.; Ma, X.; Yang, G.; Xu, D.; Liu, X.; Wang, G.; Chen, Z.; et al. Fabrication of hydroxyapatite/hydrophilic graphene composites and their modulation to cell behavior toward bone reconstruction engineering. Colloids Surf. B Biointerfaces 2019, 173, 512-520. [CrossRef]

19. Zheng, P.; Hu, X.; Lou, Y.; Tang, K. A Rabbit Model of Osteochondral Regeneration Using Three-Dimensional Printed Polycaprolactone-Hydroxyapatite Scaffolds Coated with Umbilical Cord Blood Mesenchymal Stem Cells and Chondrocytes. Med. Sci. Monit. 2019, 25, 7361-7369. [CrossRef]

20. Jeong, S.I.; Ko, E.K.; Yum, J.; Jung, C.H.; Lee, Y.M.; Shin, H. Nanofibrous poly(lactic acid)/hydroxyapatite composite scaffolds for guided tissue regeneration. Macromol. Biosci 2008, 8, 328-338. [CrossRef]

21. Sena, L.A.; Caraballo, M.M.; Rossi, A.M.; Soares, G.A. Synthesis and characterization of biocomposites with different hydroxyapatite-collagen ratios. J. Mater. Sci. Mater. Med. 2009, 20, 2395-2400. [CrossRef] [PubMed]

22. Wei, J.; Li, Y. Tissue engineering scaffold material of nano-apatite crystals and polyamide composite. Eur. Polym. J. 2004, 40, 509-515.

23. Ciapetti, G.; Ambrosio, L.; Savarino, L.; Granchi, D.; Cenni, E.; Baldini, N.; Pagani, S.; Guizzardi, S.; Causa, F.; Giunti, A. Osteoblast growth and function in porous poly epsilon -caprolactone matrices for bone repair: A preliminary study. Biomaterials 2003, 24, 3815-3824. [CrossRef]

24. Zhang, R.; Ma, P.X. Poly(alpha-hydroxyl acids)/hydroxyapatite porous composites for bone-tissue engineering. I. Preparation and morphology. J. Biomed. Mater. Res. 1999, 44, 446-455. [CrossRef]

25. De Santis, R.; Russo, A.; Gloria, A.; D’Amora, U.; Russo, T.; Panseri, S.; Sandri, M.; Tampieri, A.; Marcacci, M.; Dediu, V.A.; et al. Towards the Design of 3D Fiber-Deposited Poly(-caprolactone)/Iron-Doped Hydroxyapatite Nanocomposite Magnetic Scaffolds for Bone Regeneration. J. Biomed. Nanotechnol. 2015, 11, 1236-1246. [CrossRef] [PubMed]

26. Shi, S.F.; Jia, J.F.; Guo, X.K.; Zhao, Y.P.; Chen, D.S.; Guo, Y.Y.; Cheng, T.; Zhang, X.L. Biocompatibility of chitosan-coated iron oxide nanoparticles with osteoblast cells. Int. J. Nanomed. 2012, 7, 5593-5602.

27. Hung, H.S.; Chu, M.Y.; Lin, C.H.; Wu, C.C.; Hsu, S.H. Mediation of the migration of endothelial cells and fibroblasts on polyurethane nanocomposites by the activation of integrin-focal adhesion kinase signaling. J. Biomed. Mater. Res. A 2012, 100, 26-37. [CrossRef]

28. Morouço, P.; Biscaia, S.; Viana, T.; Franco, M.; Malça, C.; Mateus, A.; Moura, C.; Ferreira, F.C.; Mitchell, G.; Alves, N.M. Fabrication of Poly (-caprolactone) Scaffolds Reinforced with Cellulose Nanofibers, with and without the Addition of Hydroxyapatite Nanoparticles. BioMed Res. Int. 2016, 2016, 10. [CrossRef]

29. Szcześ, A.; Hołysz, L.; Chibowski, E. Synthesis of hydroxyapatite for biomedical applications. Adv. Colloid Interface Sci. 2017, 249, 321-330. [CrossRef]

30. Demirtaş, T.T.; Irmak, G.; Gümüşderelioğlu, M. A bioprintable form of chitosan hydrogel for bone tissue engineering. Biofabrication 2017, 9, 035003. [CrossRef]

31. Chaudhuri, O.; Gu, L.; Klumpers, D.; Darnell, M.; Bencherif, S.A.; Weaver, J.C.; Huebsch, N.; Lee, H.P.; Lippens, E.; Duda, G.N.; et al. Hydrogels with tunable stress relaxation regulate stem cell fate and activity. Nat. Mater. 2016, 15, 326-334. [CrossRef] [PubMed]

32. Katarivas Levy, G.; Ong, J.; Birch, M.A.; Justin, A.W.; Markaki, A.E. Albumin-Enriched Fibrin Hydrogel Embedded in Active Ferromagnetic Networks Improves Osteoblast Differentiation and Vascular Self-Organisation. Polymers 2019, 11, 1743. [CrossRef] [PubMed]

33. Russo, T.; D’Amora, U.; Gloria, A.; Tunesi, M.; Sandri, M.; Rodilossi, S.; Albani, D.; Forloni, G.; Giordano, C.; Cigada, A.; et al Systematic Analysis of Injectable Materials and 3D Rapid Prototyped Magnetic Scaffolds: From CNS Applications to Soft and Hard Tissue Repair/Regeneration. Procedia Eng. 2013, 59, 233-239. [CrossRef]

34. Gloria, A.; Russo, T.; D’Amora, U.; Santin, M.; De Santis, R.; Ambrosio, L. Customised multiphasic nucleus/annulus scaffold for intervertebral disc repair/regeneration. Connect. Tissue Res. 2020, 61, 152-162. [CrossRef]

35. Fu, S.; Ni, P.; Wang, B.; Chu, B.; Zheng, L.; Luo, F.; Luo, J.; Qian, Z. Injectable and thermo-sensitive PEG-PCL-PEG copolymer/collagen/n-HA hydrogel composite for guided bone regeneration. Biomaterials 2012, 33, 4801-4809. [CrossRef]

36. Kinneberg, K.R.; Nelson, A.; Stender, M.E.; Aziz, A.H.; Mozdzen, L.C.; Harley, B.A.; Bryant, S.J.; Ferguson, V.L. Reinforcement of Mono- and Bi-layer Poly(Ethylene Glycol) Hydrogels with a Fibrous Collagen Scaffold. Ann. Biomed. Eng. 2015, 43, 2618-2629. [CrossRef]

37. Qu, Y.; Wang, B.; Chu, B.; Liu, C.; Rong, X.; Chen, H.; Peng, J.; Qian, Z. Injectable and Thermosensitive Hydrogel and PDLLA Electrospun Nanofiber Membrane Composites for Guided Spinal Fusion. ACS Appl. Mater. Interfaces 2018, 10, 4462-4470. [CrossRef]

38. Gruber, R.M.; Krohn, S.; Mauth, C.; Dard, M.; Molenberg, A.; Lange, K.; Perske, C.; Schliephake, H. Mandibular reconstruction using a calcium phosphate/polyethylene glycol hydrogel carrier with BMP-2. J. Clin. Periodontol. 2014, 41, 820-826. [CrossRef]

39. Yadid, M.; Feiner, R.; Dvir, T. Gold Nanoparticle-Integrated Scaffolds for Tissue Engineering and Regenerative Medicine. Nano Lett. 2019, 19, 2198-2206. [CrossRef]

40. Hu, M.; Chen, J.; Li, Z.Y.; Au, L.; Hartland, G.V.; Li, X.; Marquez, M.; Xia, Y. Gold nanostructures: Engineering their plasmonic properties for biomedical applications. Chem Soc. Rev. 2006, 35, 1084-1094. [CrossRef] 
41. Vial, S.; Reis, R.L.; Oliveira, J.M. Recent advances using gold nanoparticles as a promising multimodal tool for tissue engineering and regenerative medicine. Curr. Opin. Solid State Mater. Sci. 2017, 21, 92-112. [CrossRef]

42. Hung, H.S.; Wu, C.C.; Chien, S.; Hsu, S.H. The behavior of endothelial cells on polyurethane nanocomposites and the associated signaling pathways. Biomaterials 2009, 30, 1502-1511. [CrossRef]

43. Hung, H.S.; Tang, C.M.; Lin, C.H.; Lin, S.Z.; Chu, M.Y.; Sun, W.S.; Kao, W.C.; Hsien-Hsu, H.; Huang, C.Y.; Hsu, S.H. Biocompatibility and favorable response of mesenchymal stem cells on fibronectin-gold nanocomposites. PLoS ONE 2013, 8, e65738. [CrossRef] [PubMed]

44. Hung, H.S.; Chang, C.H.; Chang, C.J.; Tang, C.M.; Kao, W.C.; Lin, S.Z.; Hsieh, H.H.; Chu, M.Y.; Sun, W.S.; Hsu, S.H. In vitro study of a novel nanogold-collagen composite to enhance the mesenchymal stem cell behavior for vascular regeneration. PLoS ONE 2014, 9, e104019. [CrossRef]

45. Khosravi, A.; Ghasemi-Mobarakeh, L.; Mollahosseini, H.; Ajalloueian, F.; Masoudi Rad, M.; Norouzi, M.-R.; Sami Jokandan, M.; Khoddami, A.; Chronakis, I.S. Immobilization of silk fibroin on the surface of PCL nanofibrous scaffolds for tissue engineering applications. J. Appl. Polym. Sci. 2018, 135, 46684. [CrossRef]

46. Lee, D.; Heo, D.N.; Lee, S.J.; Heo, M.; Kim, J.; Choi, S.; Park, H.-K.; Park, Y.G.; Lim, H.-N.; Kwon, I.K. Poly(lactide-co-glycolide) nanofibrous scaffolds chemically coated with gold-nanoparticles as osteoinductive agents for osteogenesis. Appl. Surf. Sci. 2018, 432, 300-307. [CrossRef]

47. Yi, C.; Liu, D.; Fong, C.C.; Zhang, J.; Yang, M. Gold nanoparticles promote osteogenic differentiation of mesenchymal stem cells through p38 MAPK pathway. ACS Nano 2010, 4, 6439-6448. [CrossRef]

48. Zhou, J.; Zhang, Y.; Li, L.; Fu, H.; Yang, W.; Yan, F. Human $\beta$-defensin 3-combined gold nanoparticles for enhancement of osteogenic differentiation of human periodontal ligament cells in inflammatory microenvironments. Int. J. Nanomed. 2018, 13, 555-567. [CrossRef]

49. Ross, R.D.; Roeder, R.K. Binding affinity of surface functionalized gold nanoparticles to hydroxyapatite. J. Biomed. Mater. Res. A 2011, 99, 58-66. [CrossRef]

50. Lee, S.J.; Lee, H.J.; Kim, S.Y.; Seok, J.M.; Lee, J.H.; Kim, W.D.; Kwon, I.K.; Park, S.Y.; Park, S.A. In situ gold nanoparticle growth on polydopamine-coated 3D-printed scaffolds improves osteogenic differentiation for bone tissue engineering applications: In vitro and in vivo studies. Nanoscale 2018, 10, 15447-15453. [CrossRef]

51. Borciani, G.; Montalbano, G.; Baldini, N.; Cerqueni, G.; Vitale-Brovarone, C.; Ciapetti, G. Co-culture systems of osteoblasts and osteoclasts: Simulating in vitro bone remodeling in regenerative approaches. Acta Biomater. 2020, 108, 22-45. [CrossRef] [PubMed]

52. Safari, B.; Aghanejad, A.; Roshangar, L.; Davaran, S. Osteogenic effects of the bioactive small molecules and minerals in the scaffold-based bone tissue engineering. Colloids Surf. B Biointerfaces 2020, 198, 111462. [CrossRef] [PubMed]

53. Editorial Office, I. International Journal of Molecular Science 2018 Best Paper Award. Int. J. Mol. Sci. 2018, $19,3694$.

54. Sugiura, F.; Kitoh, H.; Ishiguro, N. Osteogenic potential of rat mesenchymal stem cells after several passages. Biochem. Biophys. Res. Commun. 2004, 316, 233-239. [CrossRef] [PubMed]

55. Ishikawa, H.; Kitoh, H.; Sugiura, F.; Ishiguro, N. The effect of recombinant human bone morphogenetic protein-2 on the osteogenic potential of rat mesenchymal stem cells after several passages. Acta Orthop. 2007, 78, 285-292. [CrossRef] [PubMed]

56. Frassica, M.T.; Jones, S.K.; Diaz-Rodriguez, P.; Hahn, M.S.; Grunlan, M.A. Incorporation of a silicon-based polymer to PEG-DA templated hydrogel scaffolds for bioactivity and osteoinductivity. Acta Biomater. 2019, 99, 100-109. [CrossRef]

57. Kutikov, A.B.; Skelly, J.D.; Ayers, D.C.; Song, J. Templated repair of long bone defects in rats with bioactive spiral-wrapped electrospun amphiphilic polymer/hydroxyapatite scaffolds. ACS Appl. Mater. Interfaces 2015, 7, 4890-4901. [CrossRef]

58. Gao, G.; Schilling, A.F.; Yonezawa, T.; Wang, J.; Dai, G.; Cui, X. Bioactive nanoparticles stimulate bone tissue formation in bioprinted three-dimensional scaffold and human mesenchymal stem cells. Biotechnol. J. 2014, 9, 1304-1311. [CrossRef]

59. Hsieh, S.C.; Chen, H.J.; Hsu, S.H.; Yang, Y.C.; Tang, C.M.; Chu, M.Y.; Lin, P.Y.; Fu, R.H.; Kung, M.L.; Chen, Y.W.; et al. Prominent Vascularization Capacity of Mesenchymal Stem Cells in Collagen-Gold Nanocomposites. ACS Appl. Mater. Interfaces 2016, 8, 28982-29000. [CrossRef]

60. Hsu, S.H.; Tang, C.M.; Tseng, H.J. Biocompatibility of poly (ether) urethane-gold nanocomposites. J. Biomed. Mater. Res. Part A 2006, 79, 759-770. [CrossRef]

61. Ding, D.C.; Shyu, W.C.; Chiang, M.F.; Lin, S.Z.; Chang, Y.C.; Wang, H.J.; Su, C.Y.; Li, H. Enhancement of neuroplasticity through upregulation of beta1-integrin in human umbilical cord-derived stromal cell implanted stroke model. Neurobiol. Dis. 2007, 27, 339-353. [CrossRef] [PubMed]

62. Ahmed, S.; Ahmed, N.; Rungatscher, A.; Linardi, D.; Kulsoom, B.; Innamorati, G.; Meo, S.A.; Gebrie, M.A.; Mani, R.; Merigo, F.; et al. Cocoa Flavonoids Reduce Inflammation and Oxidative Stress in a Myocardial Ischemia-Reperfusion Experimental Model. Antioxidants 2020, 9, 167. [CrossRef]

63. Namba, T.; Koike, H.; Murakami, K.; Aoki, M.; Makino, H.; Hashiya, N.; Ogihara, T.; Kaneda, Y.; Kohno, M.; Morishita, R. Angiogenesis induced by endothelial nitric oxide synthase gene through vascular endothelial growth factor expression in a rat hindlimb ischemia model. Circulation 2003, 108, 2250-2257. [CrossRef] [PubMed]

64. Zikherman, J.; Doan, K.; Parameswaran, R.; Raschke, W.; Weiss, A. Quantitative differences in CD45 expression unmask functions for CD45 in B-cell development, tolerance, and survival. Proc. Natl. Acad. Sci. USA 2012, 109, E3-E12. [CrossRef] 
65. Hung, H.S.; Yang, Y.C.; Lin, Y.C.; Lin, S.Z.; Kao, W.C.; Hsieh, H.H.; Chu, M.Y.; Fu, R.H.; Hsu, S.H. Regulation of human endothelial progenitor cell maturation by polyurethane nanocomposites. Biomaterials 2014, 35, 6810-6821. [CrossRef] [PubMed]

66. Chen, Y.W.; Hsieh, S.C.; Yang, Y.C.; Hsu, S.H.; Kung, M.L.; Lin, P.Y.; Hsieh, H.H.; Lin, C.H.; Tang, C.M.; Hung, H.S. Functional engineered mesenchymal stem cells with fibronectin-gold composite coated catheters for vascular tissue regeneration. Nanomedicine 2018, 14, 699-711. [CrossRef] [PubMed]

67. Zhu, W.; Chu, C.; Kuddannaya, S.; Yuan, Y.; Walczak, P.; Singh, A.; Song, X.; Bulte, J.W.M. In Vivo Imaging of Composite Hydrogel Scaffold Degradation Using CEST MRI and Two-Color NIR Imaging. Adv. Funct. Mater. 2019, 29, 1903753. [CrossRef]

68. Ding, J.; Zhang, J.; Li, J.; Li, D.; Xiao, C.; Xiao, H.; Yang, H.; Zhuang, X.; Chen, X. Electrospun polymer biomaterials. Prog. Polym. Sci. 2019, 90, 1-34. [CrossRef]

69. Oteri, G.; Pizzino, G.; Pisano, M.; Peditto, M.; Squadrito, F.; Bitto, A. Polyethylene glycol formulations show different soft tissue remodeling and angiogenesis features. Tissue Eng. Part. A 2015, 21, 580-585. [CrossRef]

70. Cai, L.; Wang, Q.; Gu, C.; Wu, J.; Wang, J.; Kang, N.; Hu, J.; Xie, F.; Yan, L.; Liu, X.; et al. Vascular and micro-environmental influences on MSCS-coral hydroxyapatite construct-based bone tissue engineering. Biomaterials 2011, 32, 8497-8505. [CrossRef]

71. Xia, Y.; Sun, J.; Zhao, L.; Zhang, F.; Liang, X.J.; Guo, Y.; Weir, M.D.; Reynolds, M.A.; Gu, N.; Xu, H.H.K. Magnetic field and nano-scaffolds with stem cells to enhance bone regeneration. Biomaterials 2018, 183, 151-170. [CrossRef]

72. Ito, T.; Sasaki, M.; Taguchi, T. Enhanced ALP activity of MG63 cells cultured on hydroxyapatite-poly(ethylene glycol) hydrogel composites prepared using EDTA-OH. Biomed. Mater. 2015, 10, 015025. [CrossRef]

73. Mahmoud, N.S.; Ahmed, H.H.; Mohamed, M.R.; Amr, K.S.; Aglan, H.A.; Ali, M.A.M.; Tantawy, M.A. Role of nanoparticles in osteogenic differentiation of bone marrow mesenchymal stem cells. Cytotechnology 2020, 72, 1-22. [CrossRef] [PubMed]

74. Liang, H.; Xu, X.; Feng, X.; Ma, L.; Deng, X.; Wu, S.; Liu, X.; Yang, C. Gold nanoparticles-loaded hydroxyapatite composites guide osteogenic differentiation of human mesenchymal stem cells through Wnt/ $\beta$-catenin signaling pathway. Int. J. Nanomed. 2019, 14, 6151-6163. [CrossRef]

75. Tetè, S.; Zara, S.; Vinci, R.; Zizzari, V.; Di Tore, U.; Mastrangelo, F.; Cataldi, A.; Gherlone, E. Vascular endothelial growth factor and e-nitric oxide synthase-mediated regenerative response occurring upon autologous and heterologous bone grafts. Int. J. Immunopathol. Pharm. 2009, 22, 1105-1116. [CrossRef] [PubMed]

76. Li, X.; Wang, Y.; Wang, Z.; Qi, Y.; Li, L.; Zhang, P.; Chen, X.; Huang, Y. Composite PLA/PEG/nHA/Dexamethasone Scaffold Prepared by 3D Printing for Bone Regeneration. Macromol. Biosci. 2018, 18, e1800068. [CrossRef] [PubMed]

77. Malpuech-Brugère, C.; Nowacki, W.; Daveau, M.; Gueux, E.; Linard, C.; Rock, E.; Lebreton, J.; Mazur, A.; Rayssiguier, Y. Inflammatory response following acute magnesium deficiency in the rat. Biochim. Biophys. Acta 2000, 1501, 91-98. [CrossRef]

78. Mak, I.T.; Dickens, B.F.; Komarov, A.M.; Wagner, T.L.; Phillips, T.M.; Weglicki, W.B. Activation of the neutrophil and loss of plasma glutathione during Mg-deficiency-modulation by nitric oxide synthase inhibition. Mol. Cell Biochem. 1997, 176, 35-39. [CrossRef]

79. Yu, L.; Rowe, D.W.; Perera, I.P.; Zhang, J.; Suib, S.L.; Xin, X.; Wei, M. Intrafibrillar Mineralized Collagen-Hydroxyapatite-Based Scaffolds for Bone Regeneration. ACS Appl. Mater. Interfaces 2020, 12, 18235-18249. [CrossRef]

80. Heo, D.N.; Ko, W.K.; Lee, H.R.; Lee, S.J.; Lee, D.; Um, S.H.; Lee, J.H.; Woo, Y.H.; Zhang, L.G.; Lee, D.W.; et al. Titanium dental implants surface-immobilized with gold nanoparticles as osteoinductive agents for rapid osseointegration. J. Colloid Interface Sci. 2016, 469, 129-137. [CrossRef] 